\title{
Revised GLE database: Fluences of solar energetic particles as measured by the neutron-monitor network since 1956^
}

\author{
I. Usoskin ${ }^{1,2}$, S. Koldobskiy ${ }^{3}$, G. A. Kovaltsov ${ }^{4}$, A. Gil ${ }^{5,6}$, I. Usoskina ${ }^{2}$, T. Willamo ${ }^{7}$, and A. Ibragimov ${ }^{8}$ \\ 1 Space Physics and Astronomy Research Unit, University of Oulu, Oulu, Finland \\ e-mail: Ilya.Usoskin@oulu.fi \\ 2 Sodankylä Geophysical Observatory, University of Oulu, Oulu, Finland \\ 3 National Research Nuclear University MEPhI, Moscow, Russia \\ 4 A.F. Ioffe Physical-Technical Institute of Russian Academy of Sciences, St. Petersburg, Russia \\ 5 Siedlce University, Faculty of Exact and Natural Sciences, Institute of Mathematics, Siedlce, Poland \\ 6 Space Research Centre of Polish Academy of Sciences, Warsaw, Poland \\ 7 Department of Physics, University of Helsinki, Helsinki, Finland \\ ${ }^{8}$ Independent Researcher, Helsinki, Finland
}

Received 27 April 2020 / Accepted 1 June 2020

\begin{abstract}
Aims. Continuous measurements of ground-based neutron monitors (NMs) form the main data source for studying high-energy highintensity solar energetic particle (SEP) events that are called ground-level enhancements (GLEs). All available data are collected in the International GLE Database (IGLED), which provides formal NM count-rate increases above the constant pre-increase level which is due to galactic cosmic rays (GCR). This data set is used to reconstruct the energy spectra of GLE events. However, the assumption of a constant GCR background level throughout GLE events is often invalid. Here we thoroughly revise the IGLED and provide a data set of detrended NM count-rate increases that accounts for the variable GCR background.

Methods. The formal GLE count-rate increases were corrected for the variable GCR background, which may vary significantly during GLE events. The corresponding integral omnidirectional fluences of SEPs were reconstructed for all GLEs with sufficient strength from the detrended data using the effective rigidity method.

Results. The database of the detrended NM count rate is revised for GLE events since 1956. Integral omnidirectional fluences were estimated for 58 GLE events and parametrised for 52 sufficiently strong events using the modified Ellison-Ramaty spectral shape. Conclusions. The IGLED was revised to account for the variable GCR background. Integral omnidirectional fluences reconstructed for most of GLE events were added to IGLED. This forms the basis for more precise studies of parameters of SEP events and thus for solar and space physics.
\end{abstract}

Key words. Sun: particle emission - catalogs - Sun: flares - solar-terrestrial relations

\section{Introduction}

Galactic cosmic rays (GCRs) are always present in the vicinity of Earth, and their flux is modulated in the course of the 11 -year solar cycle by variable solar magnetic activity (see e.g. Potgieter 2013). A standard instrument for monitoring GCR variability is the worldwide network of ground-based neutron monitors (NMs), which has been in continuous operation since the early 1950s (Stoker 2009; Simpson 2000). The NM network included in different years from two to one hundred individual stations that were and are located around the globe, from equatorial regions to the Central Antarctic plateau. Currently, about 50 NMs are in operation and monitor CR variability. Their data are stored in several archives, such as the World Data Centre for Cosmic Rays (WDC CR ${ }^{1}$ ), the Neutron Monitor Database $\left(\mathrm{NMDB}^{2}\right)$, and the IZMIRAN database ${ }^{3}$.

\footnotetext{
* The revised fluences are only available at the CDS via anonymous ftp to cdsarc.u-strasbg.fr $(130.79 .128 .5)$ or via http: //cdsarc.u-strasbg.fr/viz-bin/cat/J/A+A/640/A17

1 http://cidas.isee.nagoya-u.ac.jp/WDCCR/

2 http://nmdb.eu

http://cro.izmiran.ru/common/links.htm
}

In addition to the GCR variability, the flux of energetic particles near Earth can be greatly enhanced during sporadic solar eruptive events (Vainio et al. 2009; Desai \& Giacalone 2016; Klein \& Dalla 2017), such as flares and/or coronal mass ejections (CMEs). These events are known as solar energetic particle (SEP) events. Hundreds of such events occur in every solar cycle, but they can usually only be detected in open space, beyond the protective shield of the Earth's atmosphere and magnetosphere. Occasionally, SEP events may have a sufficiently hard spectrum and high intensity to be recorded by the ground-based NMs (Shea \& Smart 2012; Aschwanden 2012). This class of strong SEP events is known as ground-level enhancements (GLEs) of the NM count rates. At present, 72 such GLE events are known and numbered consecutively since GLE 1 in February 1942. The first 4 GLEs were recorded by ground-based ionisation chambers (Forbush 1946), and their parameters are not defined, while events starting from event 5 (23 February 1956) were recorded by NMs and form the main object of this study. All available information on the count rates of NMs during the GLEs from event 5 onwards is collected in the International GLE Database $\left(\right.$ IGLED $^{4}$ ), which was originally created by Louise Gentile (from

\footnotetext{
4 https://gle.oulu.fi
} 

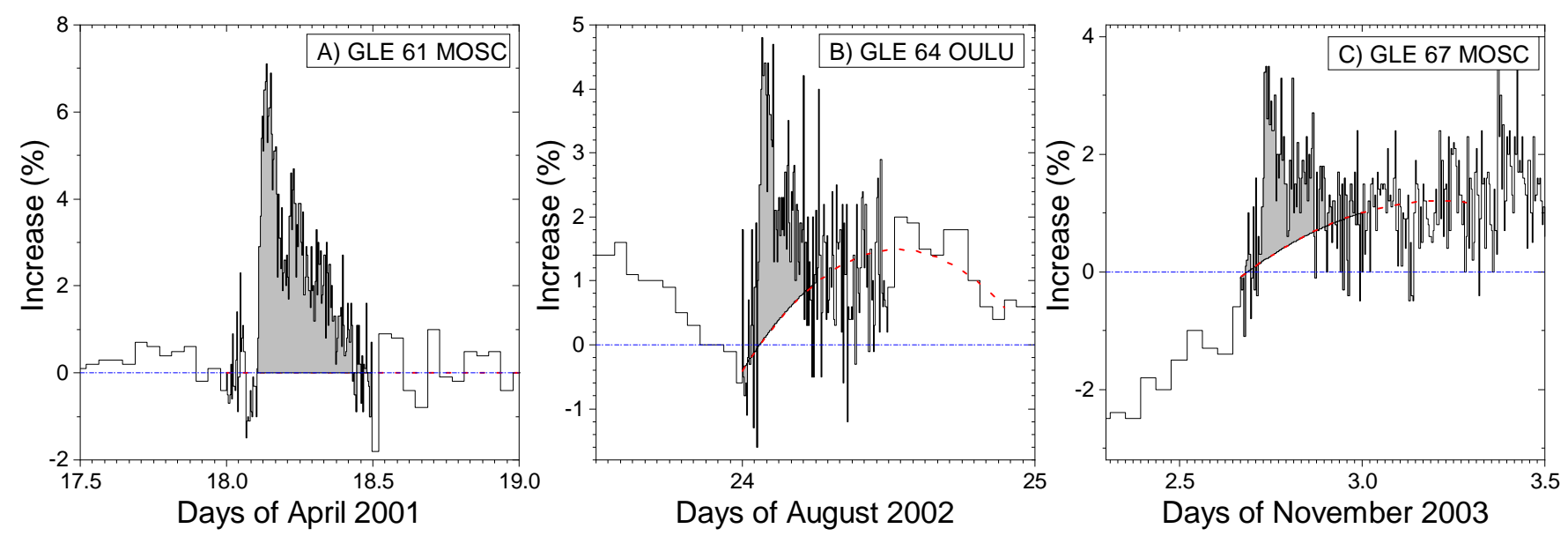

Fig. 1. Examples of GLE time profiles on the background of different baselines: quiet period (panel a), diurnal waves (panel b), and recovery after a Forbush decrease (panel c). The dash-dotted blue lines indicate the formal baseline, corresponding to $N_{0}$, the dashed red lines depict the variable baseline $N_{\mathrm{b}}$, and the grey shading indicates the increase $I_{\mathrm{d}}$ above it. The data were obtained from the International GLE Database (https://gle.oulu.fi), and GLE number and NM name are given in the legend.

Emmanuel College, Boston USA), was developed by Margareth A. Shea and Don Smart (Air Force Geophysics Laboratory, USA), and was later continued by Marc Duldig (University of Tasmania, Australia). Since 2014, it is maintained by the University of Oulu (Finland). The database includes 2824 individual records, each being an ASCII-text file (see Appendix A) containing the header with meta-data; the body with the time-stamped readings of the uncorrected count rates, barometric pressure, corrected count rates, and the formal relative count rate increases; and a footer with comments. The formal relative GLE increase $I_{\mathrm{f}}$ is given in percent with respect to the constant pre-increase level due to GCR, $N_{o}$ :

$I_{\mathrm{f}}(t)=\frac{N(t)-N_{o}}{N_{o}} \cdot 100 \%$,

where $N_{o}$ is defined as the average pressure-corrected count rate of the NM during the pre-increase time interval (one or two full hours before the GLE onset, as specified in the file headers), which is the same for all NMs for a given GLE. This data set forms the basis for studies of GLE events. This study is important in different respects, including both astrophysical studies (physics of solar eruptive events, acceleration and transport of energetic particles, estimates of their spectral and anisotropy parameters) and practical applications (e.g. assessment of radiation doses and ionisation in the atmosphere and in space). For the former, the time profile of the GLE signal as recorded by different NMs is required, while for the latter, the event-integrated fluence is sufficient. Thus, it is crucially important that the IGLED data set, which provides the official data source for GLE data, is verified and correct.

We here critically revise the existing database against the explicit assumption that the baseline level $N_{o}$ is constant during a GLE event. We show that this assumption is not valid in most cases, because the GCR is essentially variable. In addition to the relatively slow solar-cycle modulation, GCRs sometimes experience short-time variability due to interplanetary transients and local anisotropy (e.g. Dorman 2004; Gil et al. 2018). We recompute the relative increases by applying time-variable baselines and updated the IGLED correspondingly. We also re-assess the event-integrated fluences of GLEs and reconstruct their rigidity spectra above $1 \mathrm{GV}$.

\section{Revision of the IGLED data}

The official IGLED provides formal increases of NM count rates above the constant pre-increase background $N_{o}$, which is called the baseline (see Fig. 1). The baseline is considered to be due to the GCR background, and the increase above it is assumed to be caused by SEP (Eq. (1)). Some examples of $I_{\mathrm{f}}$ GLE time profiles are shown in Fig. 1.

This approach explicitly assumes that the GCR background does not change significantly during the GLE event. Sometimes, this assumption works well, as illustrated in Fig. 1a: for GLE event 61 on 18 April 2001, as recorded by the Moscow NM, the baseline was fairly stable and remained at the same level after the event as before it. However, the assumption of baseline constancy is not valid in many cases. Figure 1b depicts a case when the event (GLE 64 on 24 August 2002, as recorded by the Oulu $\mathrm{NM}$ ) occurred on the background of a diurnal wave caused by the local GCR anisotropy. Although the amplitude of the wave is not large, $\approx 1 \%$, it may strongly distort the GLE signal for weak events. Figure 1c illustrates another typical situation (shown for GLE 67 on 2 November 2003, as recorded by the Moscow NM) when a GLE took place on a continuously changing GCR background related to a recovery phase after a Forbush decrease (a sudden suppression of the GCR caused by an interplanetary transient, which frequently is a CME-driven interplanetary shock).

Here we revise the assumption that the baseline is constant by letting it have a smooth temporal variability, and compute the detrended GLE intensity,

$I_{\mathrm{d}}(t)=\frac{N(t)-N_{\mathrm{b}}(t)}{N_{o}} \cdot 100 \%=I_{\mathrm{f}}(t)-I_{\mathrm{b}}(t)$,

where $I_{\mathrm{b}}$ is the relative variable baseline

$I_{\mathrm{b}}(t)=\frac{N_{\mathrm{b}}(t)-N_{o}}{N_{o}} \cdot 100 \%$.

For each event and each NM separately, the time profile of the baseline $I_{\mathrm{b}}$ was defined using the algorithm described below.

First, the formal increases $I_{\mathrm{f}}$ were reduced to the hourly resolution. Next, hourly data-points, corresponding to the GLE event itself, were removed, so that only the GCR variability before and after the event was considered. The hourly values were associated with the GLE either visually for NMs with a strong response 


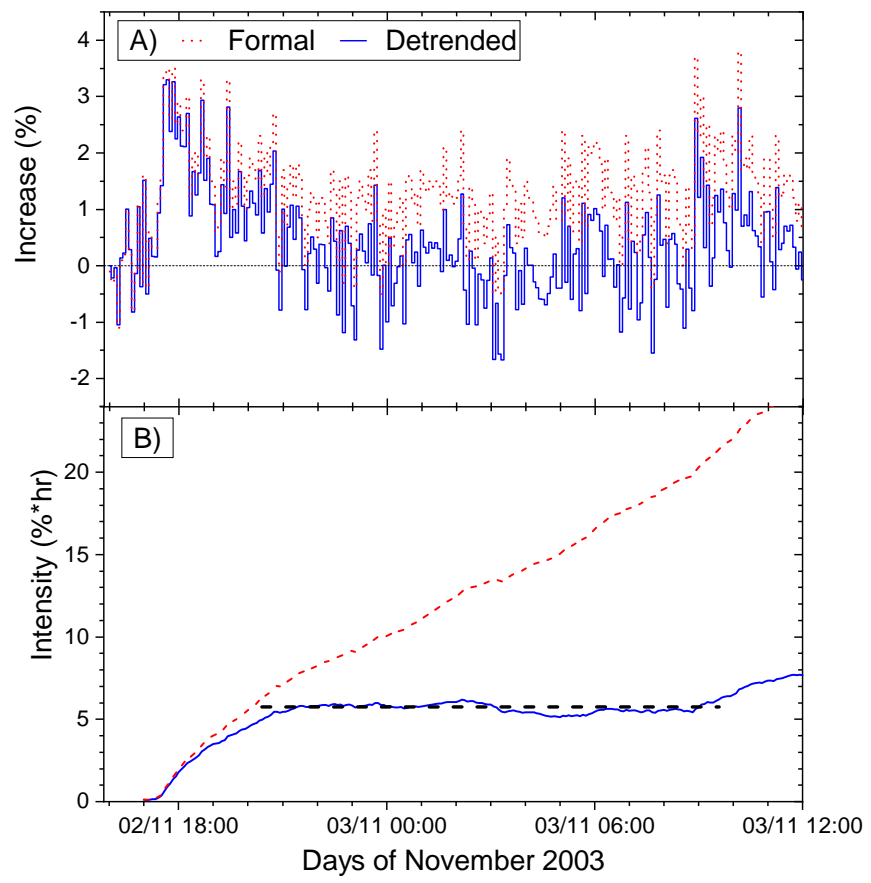

Fig. 2. Panel a: GLE time profile (GLE 67 for MOSC NM; see Fig. 1c) as provided formally above the constant baseline $\left(I_{\mathrm{f}}\right.$ is plotted as the dashed red curve), and detrended profiles for the variable baseline ( $I_{\mathrm{d}}$ is plotted as the solid blue line). Panel $b$ : cumulative intensity of GLE 67 MOSC, i.e., time integration of curves in panel $a$. The dashed black line represents the adopted integral intensity $X=5.8 \% \mathrm{~h}$.

(an apparent increase above a smooth background), or in case of higher-rigidity NMs with weak responses, based on the GLE profiles from low-rigidity NMs. The data-point(s) for the official pre-increase period (as indicated in the metadata for each NM and GLE event) were always kept for the fitting. Two to 8 hourly $I_{\mathrm{f}}$ values after the end of the apparent event were considered so that they represent the post-event background variability. These hourly values were fitted with a parabolic (second-order polynomial) curve, which was taken as the baseline $I_{\mathrm{b}}(t)$. The parabolic shape was found optimal because it provides a general balance between simplicity and realism in all analysed cases. We note that linear fits do not work in some cases, but more complicated shapes can lead to unphysical over-fitting. Examples of the fitted $I_{\mathrm{b}}$ are shown in Fig. 1. Then, the formal percentiles of the increase $I_{\mathrm{f}}$ were recomputed to $I_{\mathrm{d}}$ for the fitted baseline using Eq. (2).

The importance of accounting for the realistic baseline is illustrated in Fig. 2, where the upper panel depicts the formal $I_{\mathrm{f}}$ and the lower panel shows $I_{\mathrm{d}}$ corrected for the GLE time profiles with variable baselines. The $I_{\mathrm{f}}$ values do not return to the zero level, but remain systematically above it, at $a \approx 1 \%$ level after 21 UT on 2 November, and they even steadily increase during 3 November because the GCR level changed during and after the event. On the other hand, the detrended profile $I_{\mathrm{d}}$ returned to the zero level at about 21 UT on 2 November and remained there for about $12 \mathrm{~h}$, implying that the baseline variability was correctly accounted for.

The time profiles of corrected increases $I_{\mathrm{d}}$ for all GLEs and NMs, where they can be defined, are collected in the revised IGLED $^{5}$ and can be found at "De-trended GLE data" at the top of the web-page. Selected profiles can either be plotted or

\footnotetext{
https://gle.oulu.fi
}

downloaded as text files. The exact profiles of $I_{\mathrm{f}}$ and $I_{\mathrm{d}}$ are available for each NM and GLE in the IGLED data files (see Appendix A), while their difference defines the background profiles $I_{\mathrm{b}}$.

The bottom panel of Fig. 2 depicts the cumulative GLE intensity for the two cases, that is, for formal and detrended profiles. The cumulative event-integrated intensity $X$ is defined as the integral of the excess above the GCR background over the entire duration of the event (see the shaded area in Fig. 1) and is given here in units of percent times hours (cf. Asvestari et al. 2017). The cumulative intensity is robustly calculated for the detrended profile with only little dependence on the exact determination of the end of the event (any time between $21 \mathrm{UT}$ 2 November and 9UT 3 November fits). The mean value is $X=5.8 \% \mathrm{~h}$, and considering the uncertainties, it was rounded to $6 \% \mathrm{~h}$ in the tables available at the $\mathrm{CDS}^{6}$. In contrast, the cumulative intensity of the formal GLE profile does not have a plateau, and its value strongly depends on the exact time that is defined as the end of the event, leading to large uncertainties and unstable results. Uncertainties (full range) of the cumulative intensity $X$, corresponding to the definition of the background level $I_{\mathrm{b}}$, were conservatively estimated, based on the experience of fitting $>2000$ curves, as $0.1 \mathrm{X}$, but not smaller than $1 \% \mathrm{~h}$.

The time profiles were revised for each GLE and each NM to reach the plateau. The revised cumulative intensities are presented in tables in the CDS for each GLE and each NM.

\section{GLE integral spectra}

The high-energy part (above several hundred $\mathrm{MeV}$ ) of the SEP fluence for GLEs is evaluated in a standard way using data from the ground-based NM network (e.g. Stoker 1995; Raukunen et al. 2018). Event-integrated fluences of SEP and their energy and rigidity spectra are needed when the accumulated effects of the SEP events are assessed, such as radiation doses, atmospheric ionisation and related atmospheric response, and production of cosmogenic isotopes (e.g. Pavlov et al. 2014; Oh et al. 2012; Duderstadt et al. 2016; Melott et al. 2016; Jiggens et al. 2018; Miroshnichenko 2018; Herbst et al. 2020). The first systematic effort to estimate the SEP event-integrated omnidirectional fluence ${ }^{7} F(>R)$, where $R$ is the proton rigidity (momentum per charge) was made by Tylka \& Dietrich (2009) (updated as Raukunen et al. 2018) for GLE events 5 through 71 using NM count rates. Their analysis was based on a fitting of the count rates of individual NMs for individual GLEs, using the modelled response by applying the NM yield function by Clem \& Dorman (2000), with the prescribed power-law shape of the SEP rigidity spectrum. In this way, they determined an optimum set of the power-law parameters. This work contains two shortcomings. First, it is based on an obsolete NM yield function, which has been shown to overestimate the NM response in the lower energy range (Koldobskiy et al. 2019a) and thus to underestimate the SEP flux. As shown by Koldobskiy et al. (2019b) based on analyses of GLEs 69 and 71, the approach by Raukunen et al. (2018) may significantly underestimate the high-energy part of the SEP fluence. Second, this approach is parametric because it is based on finding best-fit parameters of

\footnotetext{
6 Strasbourg Astronomical Data Center,
}

http: //cdsweb.u-strasbg.fr

7 The integral omnidirectional fluence $F$ (in units of $\mathrm{cm}^{-2}$ ) is related to the integral intensity $J$ in units of $\left[\mathrm{cm}^{2} \mathrm{sr}\right]^{-1}$ as $J=4 \pi \cdot F$ in the isotropic case. The often-used flux of particles through a universely flat surface $S$ (also in $\mathrm{cm}^{-2}$ ) is then related to the omnidirectional fluence as $S=F / 4$ (see e.g. Grieder 2001). 


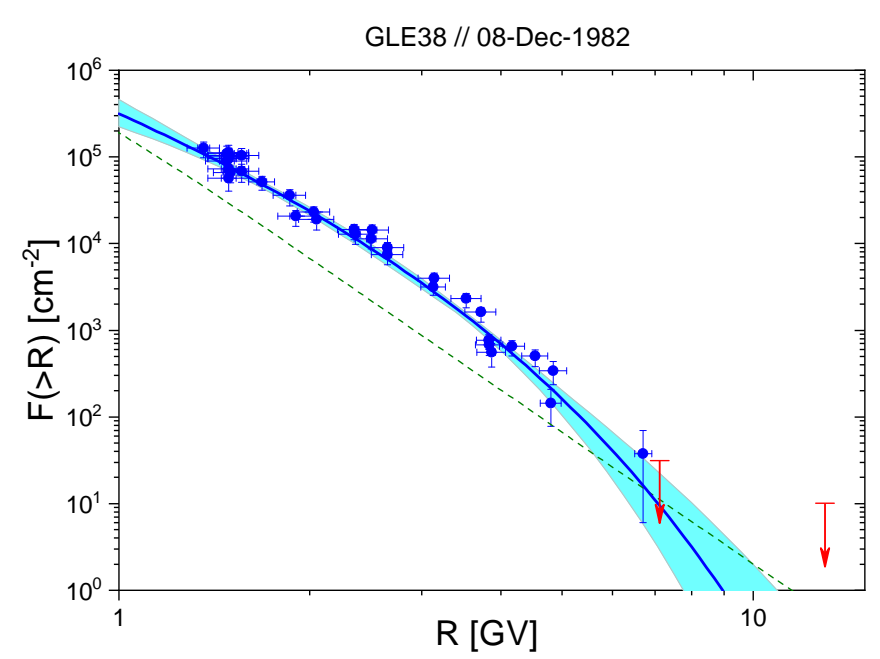

Fig. 3. Integral fluences reconstructed here for GLE 38 (8 December 1982). Blue points with error bars depict reconstructions of the integral fluence from individual NMs, as described in Sect. 4, while red arrows denote the upper limits (no statistically significant response in the NM). Error bars represent the full-range uncertainties. The thick blue curve represents the best-fit MER spectral approximation with $1 \sigma$ uncertainties (Eq. (5)). The dashed green line depicts the spectral estimate for this GLE based on the power law in rigidity fitting (Raukunen et al. 2018).

a prescribed parametric shape (power-law function in the NM energy and rigidity range).

The approach pioneered by Tylka \& Dietrich (2009) has recently been improved by Koldobskiy et al. (2019b) in the methodological sense. First, the most recent NM yield functions were used (Caballero-Lopez \& Moraal 2012; Mishev et al. 2013; Mangeard et al. 2016). Second, the newly developed nonparametric effective rigidity method (Koldobskiy et al. 2018a) was applied to reconstruct the integral spectrum. The method is based on the proportionality between the response of an NM to a GLE on one hand, and the SEP integral flux above the effective rigidity and energy on the other hand. The scaling coefficient and the corresponding value of the effective rigidity are a characteristic of the NM and not of the GLE. This enables directly relating the NM response to the integral SEP fluence for any GLE event, without a priori assumptions on its spectral shape. In this way, the spectrum is reconstructed not as a fitted prescribed model, but each NM response represents a single point on an $F-R$ diagram, where the integral fluence of SEPs for the $i$ th NM for the $j$ th GLE is defined as

$F_{i, j}\left(>R_{\mathrm{eff}, i}\right)=X_{i, j} \cdot N_{\mathrm{GCR}, j} \cdot K_{i}$,

where $K_{i}$ and $R_{\mathrm{eff}, i}$ are the scaling factor and effective rigidity of the $i$ th NM, $X_{i, j}$ is the $j$ th GLE integral intensity in $\% \mathrm{~h}$, and $N_{\mathrm{GCR}, j}$ is the baseline count rate of the ideal NM due to GCR. The scaling factor $K_{i}$ is calculated for the ideal NM at a given location (location and geomagnetic rigidity cutoff for a given date).

We here applied the method published by Koldobskiy et al. (2019b) to the revised data of the NM integral intensities for all NMs for GLEs 5 through 72 (Sect. 2). For the practical application of the method, we specify below details that are important for the reproducibility of the results:

- The method was applied to the revised GLE intensities $X_{i, j}$ as described above.

- We used the recent NM yield function (Mishev et al. 2013, 2020), validated by direct comparison with the AMS-02 and

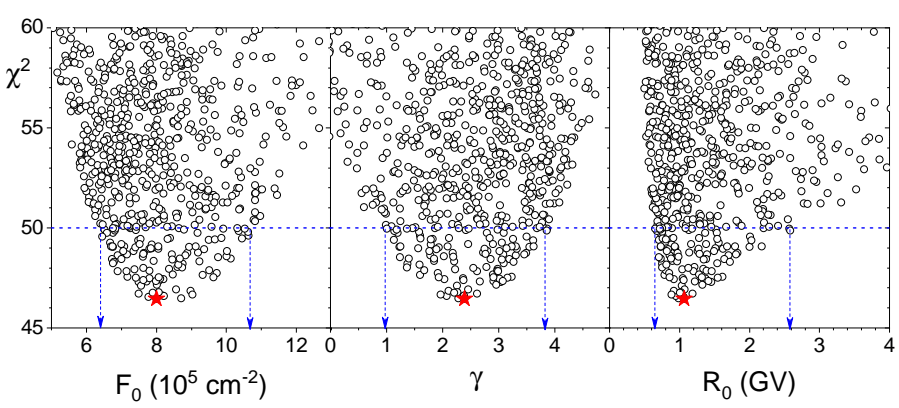

Fig. 4. Dependence of the $\chi^{2}$ value on the MER spectral parameters (Eq. (5)) for GLE 38 shown in Fig. 3. The best-fit values $\left(\chi_{\min }^{2}=46.46\right)$ are shown with the red stars. The horizontal dashed blue line corresponds to $\chi_{68}^{2}=49.99$, and the vertical blue arrows denote the $68 \%$ bounds for the parameter values.

PAMELA space-borne data for the time period between 2006 and 2017 (Koldobskiy et al. 2018b, 2019a).

- The cutoff rigidity $P_{\mathrm{c}}$ (Shea $\&$ Smart 2001) was calculated individually for each NM and each GLE, using the MAGNETOCOSMICS code (Desorgher et al. 2005, 2009).

- To assess the GCR background, we used the local interstellar spectrum (LIS) for protons according to Vos \& Potgieter (2015) and the force-field model of solar modulation (Usoskin et al. 2005, 2011). Values for the solar modulation potential were taken from Usoskin et al. (2017). Heavier GCR species $(Z>1)$ were accounted for in LIS with the constant coefficient of 0.353 with respect to protons in the number of nucleons with the same energy per nucleon, following Koldobskiy et al. (2019a).

- When a statistically significant response of an NM to a GLE was absent, the value of $1 \% \mathrm{~h}$ was used as an upper limit. Datapoints for which neither a GLE signal nor its absence can be reliably distinguished from the background variability were dismissed from the spectral reconstruction.

As a result, we reconstructed omnidirectional event-integrated fluences of SEPs for 58 GLEs, except for the weakest ones, where only polar NMs have statistically significant responses. We present the defined values of $F(>R)$ in the plots in Appendix B and in tables in CDS. GLEs 6, 7, 9, 14, 15, 17, 34, 54, 57, and 68 were too weak or have insufficient data quality to provide a basis for a robust reconstruction of the fluence.

An example of the reconstructed fluences is shown in Fig. 3 for a moderate GLE 38 (8 December 1982). Reconstructions of the integral SEP fluence based on individual NM data are shown as blue points with full-range error bars, which include both uncertainties of the effective rigidity method (Koldobskiy et al. 2018a) and those related to the definition of the background level (Sect. 2). Upper limits are defined as the absence of statistically significant response at a given NM. Points that are based on individual NMs form a smooth line that cannot be matched by a single power law but rolls down at high rigidities, as constrained by the upper limits. For comparison, the result by Raukunen et al. (2018, called R18 henceforth) for this GLE is also shown. The fluence reconstruction presented here is, first, systematically higher by a factor of up to four than that by R18 (cf. Koldobskiy et al. 2019b), and second, the fluence rolls down at high rigidities $(>5 \mathrm{GV})$ and cannot be represented by a power law. This pattern is consistent for most of the analysed events. When our points are fitted with a power law, the power law lies nearly parallel to the R18 line but is higher than their line by a factor of about two. We note that the dispersion of the points, 
I. Usoskin et al.: Revised GLE database

Table 1. Best-fit parameters of the MER spectral shape (Eq. (5)) estimated here.

\begin{tabular}{|c|c|c|c|c|c|c|c|c|c|c|}
\hline GLE number & Date & $J_{0}\left[\mathrm{~cm}^{-2}\right]$ & $\sigma J^{+}$ & $\sigma J^{-}$ & $\gamma$ & $\sigma_{\gamma}^{+}$ & $\sigma_{\gamma}^{-}$ & $R_{0}[\mathrm{GV}]$ & $\sigma_{R 0}^{+}$ & $\sigma_{R 0}^{-}$ \\
\hline 05 & $23 / 02 / 1956$ & $1.06 \mathrm{E}+8$ & $3.13 \mathrm{E}+7$ & $2.98 \mathrm{E}+7$ & 4.29 & 0.50 & 0.52 & 5.12 & 4.33 & 1.64 \\
\hline 08 & $04 / 05 / 1960$ & $9.34 \mathrm{E}+5$ & $6.42 \mathrm{E}+4$ & $2.15 E+5$ & -0.82 & 1.35 & 0.00 & 0.56 & 0.20 & 0.00 \\
\hline 10 & $12 / 11 / 1960$ & $3.03 \mathrm{E}+7$ & $4.58 \mathrm{E}+6$ & $3.75 E+6$ & 0.35 & 0.51 & 0.79 & 0.50 & 0.04 & 0.06 \\
\hline 11 & $15 / 11 / 1960$ & $1.95 \mathrm{E}+7$ & $4.10 \mathrm{E}+6$ & $4.54 \mathrm{E}+6$ & 3.89 & 1.36 & 0.90 & 1.01 & 1.23 & 0.26 \\
\hline 12 & $20 / 11 / 1960$ & $3.65 \mathrm{E}+5$ & $7.07 \mathrm{E}+5$ & $6.11 \mathrm{E}+4$ & 5.75 & 0.52 & 3.26 & $\infty$ & - & - \\
\hline 13 & $18 / 07 / 1961$ & $2.08 \mathrm{E}+6$ & $2.20 \mathrm{E}+7$ & $8.51 \mathrm{E}+5$ & 4.76 & 2.20 & 5.64 & 1.38 & $\infty$ & 1.12 \\
\hline 16 & $28 / 01 / 1967$ & $2.28 \mathrm{E}+6$ & $2.28 \mathrm{E}+5$ & $2.55 \mathrm{E}+5$ & 5.05 & 0.17 & 0.71 & 5.30 & 1.70 & 3.00 \\
\hline 18 & 29/09/1968 & - & - & - & - & - & - & - & - & - \\
\hline 19 & $18 / 11 / 1968$ & $1.23 \mathrm{E}+5$ & $5.19 \mathrm{E}+6$ & $4.29 \mathrm{E}+4$ & 5.69 & 1.36 & 8.38 & $\infty$ & - & - \\
\hline 20 & $25 / 02 / 1969$ & $2.12 \mathrm{E}+5$ & $4.10 \mathrm{E}+5$ & $6.06 \mathrm{E}+4$ & 2.15 & 1.79 & 2.94 & 0.97 & 1.56 & 0.58 \\
\hline 21 & $30 / 03 / 1969$ & $1.01 \mathrm{E}+6$ & $1.01 \mathrm{E}+5$ & $1.36 \mathrm{E}+5$ & 2.93 & 0.55 & 0.46 & 1.58 & 0.46 & 0.25 \\
\hline 22 & $24 / 01 / 1971$ & $6.17 \mathrm{E}+5$ & $4.32 \mathrm{E}+5$ & $5.83 \mathrm{E}+4$ & 4.47 & 0.54 & 1.86 & 2.05 & 1.09 & 1.31 \\
\hline 23 & 01/09/1971 & $9.11 \mathrm{E}+6$ & $1.40 \mathrm{E}+7$ & $6.83 E+6$ & 2.98 & 4.07 & 2.49 & 0.52 & $\infty$ & 0.21 \\
\hline 24 & $04 / 08 / 1972$ & $6.22 \mathrm{E}+6$ & $1.85 \mathrm{E}+7$ & $3.68 \mathrm{E}+6$ & 12.80 & 3.31 & 2.15 & $\infty$ & - & - \\
\hline 25 & $07 / 08 / 1972$ & $2.67 \mathrm{E}+5$ & $1.63 \mathrm{E}+5$ & $6.06 \mathrm{E}+4$ & 5.11 & 0.28 & 1.72 & $\infty$ & - & - \\
\hline 26 & $29 / 04 / 1973$ & $5.61 \mathrm{E}+4$ & $1.37 \mathrm{E}+5$ & $2.62 \mathrm{E}+4$ & 2.31 & 2.04 & 3.42 & 1.52 & $\infty$ & 1.10 \\
\hline 27 & $30 / 04 / 1976$ & $1.00 \mathrm{E}+5$ & $2.53 \mathrm{E}+7$ & $4.86 \mathrm{E}+4$ & 5.83 & 2.38 & 9.95 & $\infty$ & - & - \\
\hline 28 & $19 / 09 / 1977$ & $2.89 \mathrm{E}+5$ & $6.99 \mathrm{E}+5$ & $2.02 \mathrm{E}+5$ & 8.79 & 3.27 & 3.30 & $\infty$ & - & - \\
\hline 29 & $24 / 09 / 1977$ & $2.41 \mathrm{E}+5$ & $2.56 \mathrm{E}+5$ & $5.73 \mathrm{E}+4$ & 4.77 & 0.38 & 2.57 & $\infty$ & - & - \\
\hline 30 & $22 / 11 / 1977$ & $5.98 \mathrm{E}+5$ & $1.57 \mathrm{E}+5$ & $7.83 \mathrm{E}+4$ & 4.22 & 0.60 & 1.02 & 4.24 & 9.60 & 2.72 \\
\hline 31 & $07 / 05 / 1978$ & $6.26 \mathrm{E}+4$ & $1.37 \mathrm{E}+4$ & $8.44 \mathrm{E}+3$ & 1.45 & 0.70 & 0.70 & 1.83 & 1.25 & 0.51 \\
\hline 32 & 23/09/1978 & $3.12 \mathrm{E}+5$ & $8.89 \mathrm{E}+4$ & $6.61 \mathrm{E}+4$ & 4.88 & 0.28 & 0.91 & $\infty$ & - & - \\
\hline 33 & $21 / 08 / 1970$ & - & - & - & - & - & - & - & - & - \\
\hline 35 & $10 / 05 / 1981$ & - & - & - & - & - & - & - & - & - \\
\hline 36 & $12 / 10 / 1981$ & $5.78 \mathrm{E}+5$ & $1.11 \mathrm{E}+5$ & $1.11 \mathrm{E}+5$ & 3.34 & 0.78 & 0.85 & 2.73 & 10.02 & 1.23 \\
\hline 37 & $26 / 11 / 1982$ & $6.17 \mathrm{E}+4$ & $2.95 \mathrm{E}+4$ & $8.66 \mathrm{E}+3$ & 2.88 & 0.89 & 1.80 & 3.13 & 11.31 & 2.21 \\
\hline 38 & $08 / 12 / 1982$ & $8.57 \mathrm{E}+5$ & $2.23 \mathrm{E}+5$ & $2.03 E+5$ & 2.34 & 1.40 & 1.38 & 1.02 & 1.25 & 0.35 \\
\hline 39 & $16 / 02 / 1984$ & $2.10 \mathrm{E}+5$ & $1.22 \mathrm{E}+5$ & $9.35 \mathrm{E}+4$ & 1.38 & 1.98 & 1.95 & 0.87 & 2.30 & 0.37 \\
\hline 40 & $25 / 07 / 1989$ & $5.59 \mathrm{E}+4$ & $1.03 \mathrm{E}+6$ & $3.00 \mathrm{E}+4$ & 5.55 & 2.57 & 7.75 & $\infty$ & - & - \\
\hline 41 & $16 / 08 / 1989$ & $2.03 E+6$ & $7.51 \mathrm{E}+5$ & $4.30 \mathrm{E}+5$ & 2.35 & & & 0.70 & 0.13 & 0.20 \\
\hline 42 & 29/09/1989 & $1.16 \mathrm{E}+7$ & $1.16 \mathrm{E}+6$ & $1.40 \mathrm{E}+6$ & 3.54 & 0.18 & 0 & 4 & 2.09 & 1.39 \\
\hline 43 & $19 / 10 / 1989$ & $6.97 \mathrm{E}+6$ & $9.34 \mathrm{E}+5$ & $1.09 \mathrm{E}+6$ & 4.72 & 0.62 & 0.76 & 3.88 & 10.51 & 1.93 \\
\hline 44 & $22 / 10 / 1989$ & $3.02 \mathrm{E}+6$ & $3.36 \mathrm{E}+6$ & $7.44 \mathrm{E}+5$ & 1.57 & 1.04 & 2.20 & 0.55 & 0.17 & 0.20 \\
\hline 45 & $24 / 10 / 1989$ & $1.22 \mathrm{E}+7$ & $1.96 \mathrm{E}+6$ & $1.69 \mathrm{E}+6$ & 3.08 & 0.78 & 0.75 & 1.18 & 0.51 & 0.28 \\
\hline 46 & 15/11/1989 & $4.37 \mathrm{E}+4$ & $1.38 \mathrm{E}+6$ & $1.29 \mathrm{E}+4$ & 4.36 & 1.47 & 6.75 & $\infty$ & - & - \\
\hline 47 & $21 / 05 / 1990$ & $2.87 \mathrm{E}+5$ & $4.08 \mathrm{E}+4$ & $4.92 \mathrm{E}+4$ & 4.06 & 0.15 & 0.52 & $\infty$ & - & - \\
\hline 48 & $24 / 05 / 1990$ & $5.92 \mathrm{E}+5$ & $1.06 \mathrm{E}+5$ & $6.90 \mathrm{E}+4$ & 3.77 & 0.77 & 0.62 & 4.49 & $\infty$ & 2.17 \\
\hline 49 & $26 / 05 / 1990$ & $3.16 \mathrm{E}+5$ & $1.05 \mathrm{E}+5$ & $7.70 \mathrm{E}+4$ & 3.84 & 0.83 & 1.29 & 3.65 & $\infty$ & 2.40 \\
\hline 50 & $28 / 05 / 1990$ & $1.47 \mathrm{E}+5$ & $4.74 \mathrm{E}+5$ & $4.23 \mathrm{E}+4$ & 2.90 & 2.31 & 4.48 & 1.44 & $\infty$ & 1.09 \\
\hline 51 & $11 / 06 / 1991$ & $1.18 \mathrm{E}+5$ & $7.15 \mathrm{E}+4$ & $2.18 \mathrm{E}+4$ & 3.35 & 1.12 & 1.75 & 3.05 & $\infty$ & 2.15 \\
\hline 52 & $15 / 06 / 1991$ & $1.20 \mathrm{E}+6$ & $1.38 \mathrm{E}+6$ & $3.59 \mathrm{E}+5$ & 3.08 & 1.64 & 2.45 & 0.90 & 1.29 & 0.45 \\
\hline 53 & $25 / 06 / 1992$ & - & - & - & - & - & - & - & - & - \\
\hline 55 & $06 / 1$ & $1.11 \mathrm{E}+6$ & $3.89 \mathrm{E}+5$ & $3.11 \mathrm{E}+5$ & 3.17 & 1.23 & 1.51 & .37 & 2.14 & 0.63 \\
\hline 56 & 05/1998 & $5.98 \mathrm{E}+4$ & $1.84 \mathrm{E}+6$ & $2.32 \mathrm{E}+4$ & 4.79 & 2.41 & 7.10 & $\infty$ & - & - \\
\hline 58 & $24 / 08 / 1998$ & - & - & - & - & - & - & - & - & - \\
\hline 59 & $14 / 07 / 2000$ & $4.14 \mathrm{E}+6$ & $4.88 \mathrm{E}+6$ & $2.04 \mathrm{E}+6$ & 5.55 & 1.56 & 2.57 & 1.57 & $\infty$ & 1.03 \\
\hline 60 & $15 / 04 / 2001$ & $2.79 \mathrm{E}+6$ & $7.14 \mathrm{E}+5$ & $4.01 \mathrm{E}+5$ & 4.03 & 0.79 & 0.71 & 2.14 & 2.23 & 0.83 \\
\hline 61 & $18 / 04 / 2001$ & $4.94 \mathrm{E}+5$ & $7.58 \mathrm{E}+5$ & $1.84 \mathrm{E}+5$ & 1.82 & 1.90 & 2.81 & 0.96 & 3.08 & 0.55 \\
\hline 62 & $04 / 11 / 2001$ & $9.14 \mathrm{E}+6$ & $1.53 \mathrm{E}+7$ & $8.87 \mathrm{E}+6$ & -2.84 & 9.38 & 0.68 & 0.21 & $\infty$ & 0.03 \\
\hline 63 & $26 / 12 / 2001$ & - & - & - & - & - & - & - & - & - \\
\hline 64 & $24 / 08 / 2002$ & $1.45 \mathrm{E}+5$ & $1.00 \mathrm{E}+9$ & $8.22 \mathrm{E}+4$ & 6.69 & 1.35 & 12.58 & $\infty$ & - & - \\
\hline 65 & $28 / 10 / 2003$ & $2.38 \mathrm{E}+6$ & $9.58 \mathrm{E}+4$ & $6.06 \mathrm{E}+5$ & 3.87 & 1.63 & 0.25 & 1.62 & $\infty$ & 0.24 \\
\hline 66 & $29 / 10 / 2003$ & $2.82 \mathrm{E}+5$ & $8.72 \mathrm{E}+5$ & $7.64 \mathrm{E}+4$ & 5.19 & 0.76 & 3.49 & 10.55 & $\infty$ & 10.03 \\
\hline 67 & $02 / 11 / 2003$ & $1.66 \mathrm{E}+5$ & $6.63 \mathrm{E}+4$ & $3.03 E+4$ & 1.64 & 1.90 & 1.36 & 0.93 & 1.48 & 0.32 \\
\hline 69 & $20 / 01 / 2005$ & $7.87 \mathrm{E}+6$ & $1.47 \mathrm{E}+6$ & $1.11 \mathrm{E}+6$ & 4.62 & 0.68 & 0.74 & 2.21 & 2.45 & 0.79 \\
\hline 70 & $13 / 12 / 2006$ & $1.40 \mathrm{E}+6$ & $4.87 \mathrm{E}+5$ & $2.57 \mathrm{E}+5$ & 3.94 & 0.88 & 1.23 & 1.61 & 2.14 & 0.71 \\
\hline 71 & $17 / 05 / 2012$ & $1.25 \mathrm{E}+5$ & $1.19 \mathrm{E}+7$ & $5.30 \mathrm{E}+4$ & 5.69 & 1.79 & 8.98 & $\infty$ & - & - \\
\hline 72 & $10 / 09 / 2017$ & $2.88 \mathrm{E}+5$ & $2.63 E+5$ & $4.95 \mathrm{E}+4$ & 5.36 & 0.71 & 1.57 & $\infty$ & - & 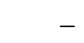 \\
\hline
\end{tabular}

Notes. Columns are the GLE number and date, parameters $J_{0}, \gamma$, and $R_{0}$ with upper $\sigma^{+}$and lower $\sigma^{-} 68 \%$ confidence intervals, defined as shown in Fig. 4. $R_{0}=\infty$ indicates that the MER shape cannot be distinguished from a pure power-law fit when the data are fit. Events with weak responses, for which the MER spectral shape cannot be obtained, are denoted with dashes. 
corresponding to polar NMs, is caused by the anisotropy of SEPs at the initial phase of the event, which is neglected here.

\section{Fitting the spectra with the modified Ellison-Ramaty shape}

The primary result of this study is a reconstruction of the omnidirectional integral fluences of SEPs during GLEs (as collected in the CDS), which forms the basic information for further analyses. Additionally, we also approximated the spectral shape of the reconstructed fluences. A single power law does not fit the reconstruction in many cases. However, we found that the shape can be approximately fitted, in all cases, by a modified EllisonRamaty (MER) spectral shape,

$F(>R)=F_{0}\left(\frac{R}{1 \mathrm{GV}}\right)^{-\gamma} \exp \left(\frac{-R}{R_{0}}\right)$,

where $F_{0}$ is a normalisation coefficient (in units of $\mathrm{cm}^{-2}$ ), $R$ is the rigidity, $\gamma$ is the spectral index, and $R_{0}$ is the roll-off rigidity. We note that $\gamma=0$ corresponds to a pure exponential case, while $R_{0}=\infty$ corresponds to a pure power law. The fit was made as described below.

We considered a GLE event with $M$ spectral points, corresponding to the registered GLE signal, and $m$ points, corresponding to the upper limits. For each iteration $l(1 \ldots N)$ and for each data point $k(1 \ldots M)$, the exact position of the reconstructed point (viz. $R_{k, l}$ and $F_{k, l}$ ) within the error bars was taken by applying the following algorithm: the value of $R_{k, l}$ was randomly and uniformly taken inside $R_{k}$ error bars; the value of $F(>R)_{k, l}$ was computed using Eq. (4), where $K_{k}$ was randomly taken inside the error bars using the uniform distribution, and $X_{k}$ was randomly taken inside the error bars, defined as $\sigma_{X, k}=\max \left[1 ; 0.1 \cdot X_{k}\right] \% \mathrm{hr}$, of the GLE intensity using the normal distribution. The rigidity interval was divided into bins of $0.4 \mathrm{GV}$ width (the first bin is $<1.3 \mathrm{GV}$ ), to avoid a bias towards more numerous polar NM for the fitting procedure. For several weak events, the bin width was reduced to $0.1 \mathrm{GV}$.

All $F_{k, l}$-vs.- $R_{k, l}$ points were dropped into the corresponding rigidity bins. For each $l$ th iteration, a set $\left(F^{*}, R^{*}\right)$ of $F_{i, j}$-vs.- $R_{i, j}$ values was randomly taken from each non-empty bin. Then, the best-fit parameters for the MER shape were found by applying a non-linear least-squares method based on minimisation of the logarithmic residual $D$ :

$D \equiv \min \left[\sum\left(\log \left(F_{\text {fit }}\left(R^{*}\right)\right)-\log \left(F^{*}\right)\right)^{2}\right]$,

where $F_{\text {fit }}$ is the value computed using Eq. (5) for the rigidity $R^{*}$. We checked that the obtained fit parameters are physically reasonable, that is, the obtained solution must not have a positive derivative anywhere in the studied rigidity range (the rigidityintegrated fluence cannot increase with $R$ ). This is quantified as $\gamma>0$, and for $\gamma<0, R_{0} \sqrt{-\gamma}<1+\gamma R_{0}$. We also required that the formal fit we found exceeded none of the $m$ upper limits. Fits that did not satisfy these conditions were discarded.

For the $l$ th set of MER parameters, the formal $\chi_{l}^{2}$ value was calculated based on all $M$ available fluence points. This set of parameters and the value of $\chi_{l}^{2}$ were recorded for the $l$ th iteration. This procedure was repeated $N=2000$ times. An example of the distribution of $\chi^{2}$ values for different sets of the fitting parameters is shown in Fig. 4, where each dot corresponds to one $l$ th set. The distribution is confined to an inverted belllike shape (non-symmetric) typical for such cases. Finally, the fit with the minimum $\chi^{2}$ value $\chi_{\min }^{2}$ was considered as the best fit

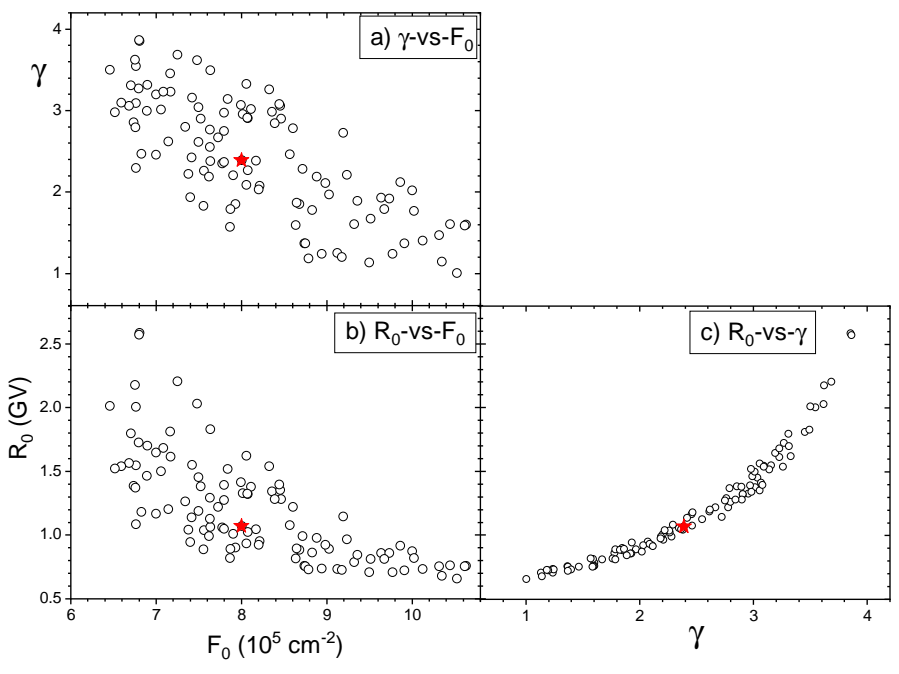

Fig. 5. Inter-relation between the best-fit (within the $68 \%$ confidence interval, viz. points below the dashed blue line in Fig. 4) parameters of the MER spectral parameters (Eq. (5)) for GLE 38. The best-fit values are shown with the red stars.

for each GLE. The $\chi_{\min }^{2}=46.46$ was found for GLE 38, which is a good fit (for 37 data points, it implies 34 degrees of freedom (d.o.f.), and $\chi^{2}=1.36$ per d.o.f.). The $1 \sigma$ range of the fitted parameters was defined (Press et al. 2007) as corresponding to $\chi^{2}<\chi_{68}^{2}=\chi_{\min }^{2}+3.53$. The obtained values (best-fit and $68 \%$ confidence intervals) enter the corresponding cells in Table 1 and are shown in Fig. 3 and in Appendix B. While the spectrum is fitted tightly in the rigidity range $1.5-5 \mathrm{GV}$, the uncertainties increase towards lower (uncertainty of a factor of two at $1 \mathrm{GV}$ ) and higher (an order of magnitude uncertainty at $10 \mathrm{GV}$ ) rigidities. It is important to note that the parameters are interrelated (see Fig. 5), with a very tight relation between $\gamma$ and $R_{0}$ (panel c). Accordingly, the parameters are not independent within their uncertainty ranges.

A full list of the best-fit MER spectral shape and their $68 \%$ confidence intervals for the analysed GLE events is given in Table 1. The corresponding curves are shown in plots in Appendix B; they are similar to Fig. 3.

A comparison between the values of SEP integral fluence reconstructed here with those from R18 is shown in Fig. 6 for several values of rigidity. A general tendency that values, obtained here, are systematically higher than those from R18 by a factor of 2-4 is visible. The difference may reach an order of magnitude for weak events $\left(F<100 \mathrm{~cm}^{-2}\right)$.

We note that the fitting considered here neglects the possible anisotropy of SEPs by implicitly assuming an isotropic distribution of energetic particles. This is similar to the assumptions of Tylka \& Dietrich (2009) and Raukunen et al. (2018).

\section{Conclusion}

The IGLED has been revised to account for the variable GCR background. The detrended GLE time profiles and the cumulative intensities were calculated for most of the GLE events. The detrended data are available in the IGLED (see Appendix A). Using these revised GLE data, we reconstructed integral omnidirectional fluences for most of the GLE events by applying the effective rigidity method (Koldobskiy et al. 2019b). The reconstructed fluences are available at the CDS. The obtained integral spectra were fitted with the modified Ellison-Ramaty spectral 

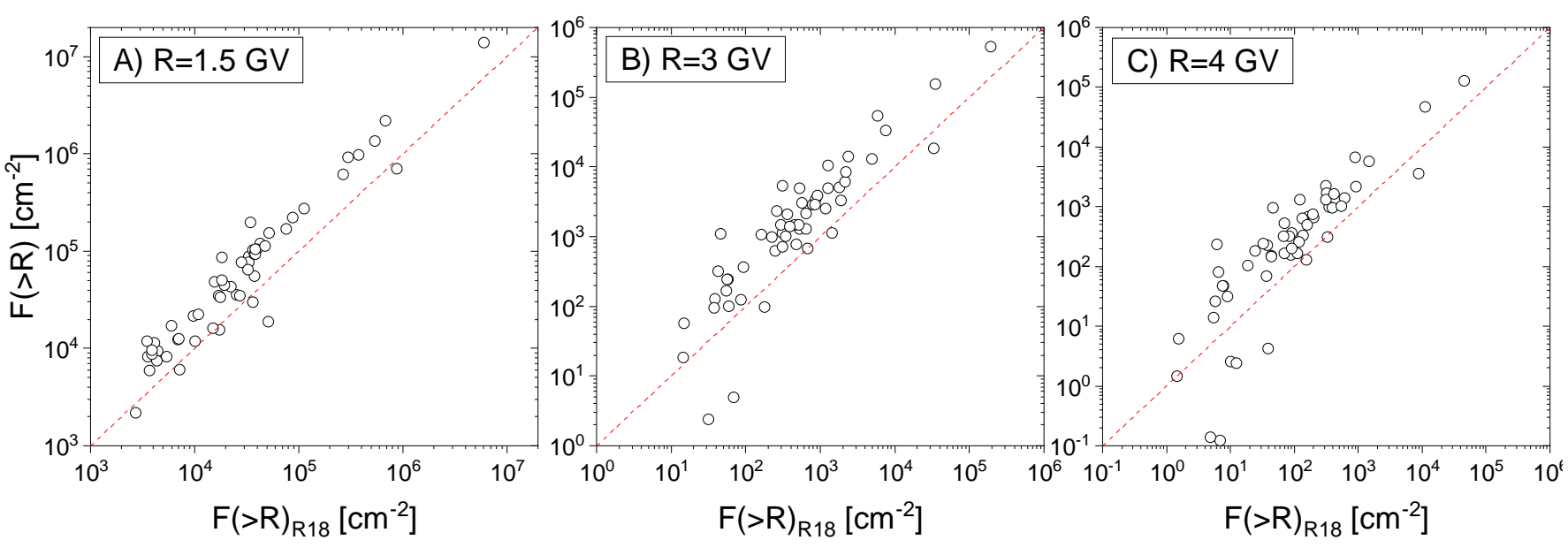

Fig. 6. Scatter plots of the integral fluences $F(>R)$ reconstructed here ( $Y$-axes) vs. those from R18 ( $X$-axes) for different values of $R$ as indicated in the legend of each panel. Dots correspond to individual GLE events, and the dashed red line denotes the diagonal of each panel.

shape (Eq. (5)), and the results of the fit are listed in Table 1. This work forms the basis for more precise studies of parameters of SEP events and, thus, for solar and space physics, including revised assessments of the SEP acceleration, transport in interplanetary space, and space weather effects.

Acknowledgements. Data of GLE recorded by NMs were obtained from the International GLE database http://gle.oulu.fi. PIs and teams of all the ground-based neutron monitors, whose data were used here, are gratefully acknowledged. DOMC/DOMB experiment is supported by IPEV and PNRA (LTCPAA project PNRA 2015/AC3). This work was partially supported by the Academy of Finland (projects No. 321882 ESPERA and No. 304435 CRIPAX), MEPhI Academic Excellence Project (contract 02.a03.21.0005) and the Polish National Science Centre (project no. 2016/22/E/HS5/00406. A part of this work (analysis of GLE spectra) was partly supported by the RSF grant No 2067-46016. The authors benefited from discussions within the ISSI International Team work (HERIOC team) and ISWAT-COSPAR S1-02 team.

\section{References}

Aschwanden, M. J. 2012, Space Sci. Rev., 171, 3

Asvestari, E., Willamo, T., Gil, A., et al. 2017, Adv. Space Res., 60, 781

Caballero-Lopez, R., \& Moraal, H. 2012, J. Geophys. Res., 117, A12103

Clem, J., \& Dorman, L. 2000, Space Sci. Rev., 93, 335

Desai, M., \& Giacalone, J. 2016, Liv. Rev. Solar Phys., 13, 3

Desorgher, L., Flückiger, E. O., Gurtner, M., Moser, M. R., \& Bütikofer, R. 2005, Int. J. Mod. Phys. A, 20, 6802

Desorgher, L., Kudela, K., Flueckiger, E., et al. 2009, Acta Geophys., 57, 75

Dorman, L. 2004, Cosmic Rays in the Earth's Atmosphere and Underground (Dordrecht: Kluwer Academic Publishers)

Duderstadt, K. A., Dibb, J. E., Schwadron, N. A., et al. 2016, J. Geophys. Res. (Atm.), 121, 2994

Forbush, S. E. 1946, Phys. Rev., 70, 771

Gil, A., Kovaltsov, G., Mikhailov, V., et al. 2018, Sol. Phys., 293, 154

Grieder, P. 2001, Cosmic Rays at Earth (Amsterdam: Elsevier Science)

Herbst, K., Banjac, S., Atri, D., \& Nordheim, T. A. 2020, A\&A, 633, A15

Jiggens, P., Heynderickx, D., Sandberg, I., et al. 2018, J. Space Weather Space Clim., 8, A31

Klein, K.-L., \& Dalla, S. 2017, Space Sci. Rev., 212, 1107

Koldobskiy, S. A., Kovaltsov, G. A., \& Usoskin, I. G. 2018a, Sol. Phys., 293, 110
Koldobskiy, S. A., Kovaltsov, G. A., \& Usoskin, I. G. 2018b, J. Geophys. Res. (Space Phys.), 123, 4479

Koldobskiy, S. A., Bindi, V., Corti, C., Kovaltsov, G. A., \& Usoskin, I. G. 2019a, J. Geophys. Res. (Space Phys.), 124, 2367

Koldobskiy, S. A., Kovaltsov, G. A., Mishev, A. L., \& Usoskin, I. G. 2019b, Sol. Phys., 294, 94

Mangeard, P.-S., Ruffolo, D., Sáiz, A., Madlee, S., \& Nutaro, T. 2016, J. Geophys. Res., 121, 7435

Melott, A., Thomas, B., Laird, C., Neuenswander, B., \& Atri, D. 2016, J. Geophys. Res.: Atm., 121, 3017

Miroshnichenko, L. I. 2018, J. Space Weather Space Clim., 8, A52

Mishev, A., Usoskin, I., \& Kovaltsov, G. 2013, J. Geophys. Res. (Space Phys.), 118,2783

Mishev, A., Koldobskiy, S., Kovaltsov, G., Gil, A., \& Usoskin, I. 2020, J. Geophys. Res. (Space Phys.), 125, e2019JA027433

Oh, S. Y., Bieber, J. W., Clem, J., et al. 2012, Space Weather, 10, S05004

Pavlov, A. K., Blinov, A. V., Vasil'ev, G. I., et al. 2014, Astron. Lett., 40, 640

Potgieter, M. 2013, Liv. Rev. Sol. Phys., 10, 3

Press, W., Teukolsky, S., Vetterling, W., \& Flannery, B. 2007, Numerical Recipes 3rd Edition: The Art of Scientific Computing, 3rd edn. (USA: Cambridge University Press)

Raukunen, O., Vainio, R., Tylka, A. J., et al. 2018, J. Space Weather Space Clim., $8, \mathrm{~A} 04$

Shea, M. A., \& Smart, D. F. 2001, Proc. 27th International Cosmic Ray Conference, Hamburg, Germany, 10, 4063

Shea, M. A., \& Smart, D. F. 2012, Space Sci. Rev., 171, 161

Simpson, J. A. 2000, Space Sci. Rev., 93, 11

Stoker, P. H. 1995, Space Sci. Rev., 73, 327

Stoker, P. H. 2009, Adv. Space Res., 44, 1081

Tylka, A., \& Dietrich, W. 2009, 31th International Cosmic Ray Conference (Lodź, Poland: Universal Academy Press), icrc0273

Usoskin, I. G., Alanko-Huotari, K., Kovaltsov, G. A., \& Mursula, K. 2005, J. Geophys. Res., 110, A12108

Usoskin, I. G., Bazilevskaya, G. A., \& Kovaltsov, G. A. 2011, J. Geophys. Res., $116, \mathrm{~A} 02104$

Usoskin, I., Ibragimov, A., Shea, M. A., \& Smart, D. F. 2015, 34th International Cosmic Ray Conference (ICRC2015), 54

Usoskin, I. G., Gil, A., Kovaltsov, G. A., Mishev, A. L., \& Mikhailov, V. V. 2017, J. Geophys. Res. (Space Phys.), 122, 3875

Vainio, R., Desorgher, L., Heynderickx, D., et al. 2009, Space Sci. Rev., 147, 187

Vos, E. E., \& Potgieter, M. S. 2015, ApJ, 815, 119 


\section{Appendix A: Download of the time profiles from IGLED}

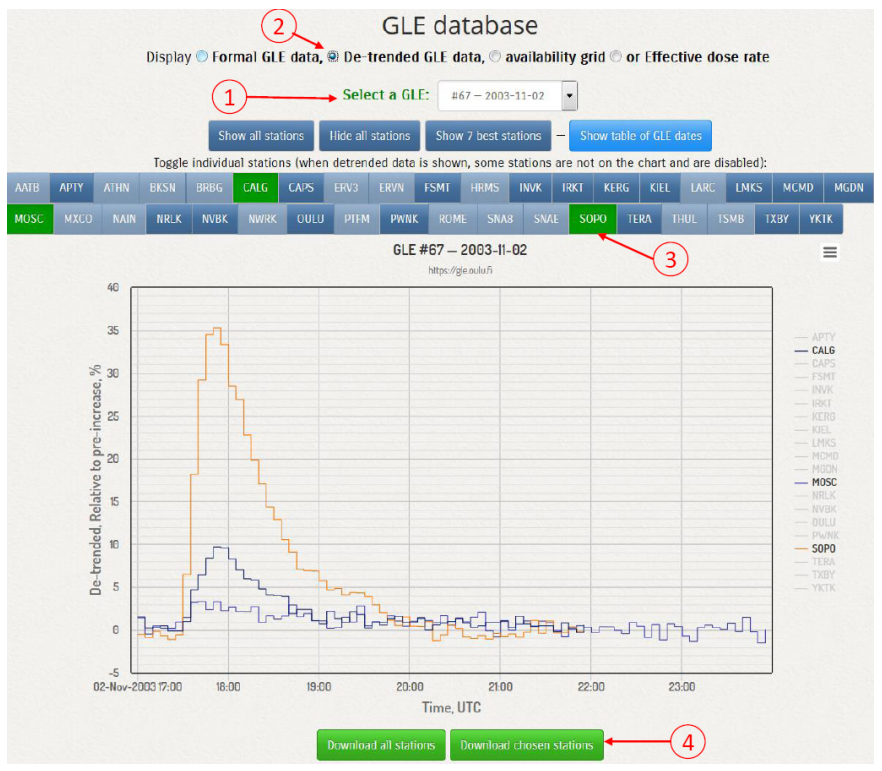

Fig. A.1. Screenshot of the IGLED. Circled numbers denote the steps for downloading GLE time profiles: (1) Selection of the GLE event; (2) selection of the detrended data; (3) selection of the NM; and (4) downloading data files.

All the raw data for the individual GLEs and NMs are collected in the IGLED and can be downloaded as text datafiles in four steps, as shown in Fig. A.1.

1. The GLE of interest can be selected from the drop-down menu as shown by (1) in Fig. A.1.

2. Formal or detrended data can be selected by radio-buttons on the top of the screen.

3. The NM(s) of interest can be selected from the list, where colour denotes the status of NMs: green, blue, and grey correspond to selected, unselected, and unavailable, respectively.

4. The large green buttons at the bottom allow downloading the data files: either all available NMs, or only the selected NMs.

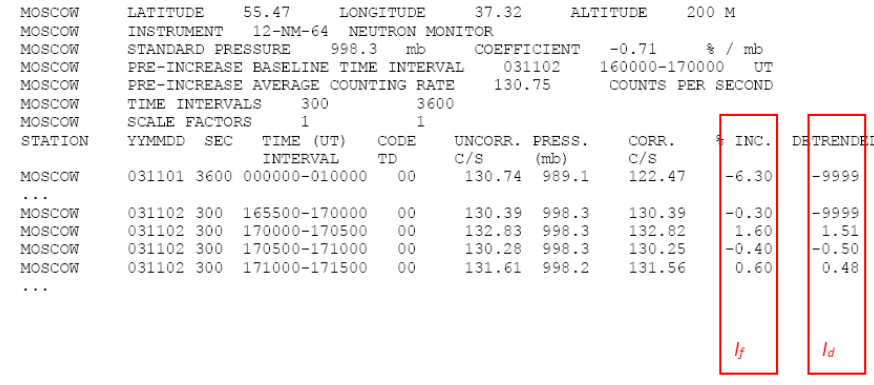

Fig. A.2. Example of the IGLED data file for MOSC NM and for GLE 67 . The file contains a standard GLE-datafile header with the housekeeping information, followed by data arranged in ten columns: NM station name; date in YYMMDD format (only the two last digits of the year are shown, thus 031102 corresponds to 2 November 2003); time interval in UT; a quality flag; uncorrected count rate; barometric pressure; corrected count rate; percentage increase $I_{\mathrm{f}}$ (Eq. (1)); and detrended $I_{\mathrm{d}}$ (Eq. (2)). -9999 denotes that no data are available.

The downloaded files are in the standard GLE format, including the header with meta-data (Usoskin et al. 2015). Figure A.2 depicts the structure of the files, where data are arranged in nine columns, following the standard GLE format, but they have one additional column corresponding to the detrended percentage increase $I_{\mathrm{d}}$ (Eq. (2)). The background profile $I_{\mathrm{b}}$ (Eq. (3)) for any NM and GLE can be defined as the difference between the 9th and the 10th columns of the file.

\section{Appendix B: Reconstructed integral fluences and spectral fits}

The following plots represent reconstructed omnidirectional integral fluences for all considered GLE events along with the MER spectral shape fits. The plots are arranged in a similar way to Fig. 3. Blue dots with error bars denote the reconstructed values of $R_{\text {eff }}$ and $F\left(>R_{\text {eff }}\right)$. The error bars represent the full-range uncertainties (see Sect. 3), and red arrows show upper limits. All data points are available digitally at the CDS. The dark blue lines represent the best-fit MER spectral shape (Eq. (5)) along with $68 \%$ confidence intervals, which are denoted as the blue shading. The fit parameters are available in Table 1 . 
GLE05 // 23 Feb 1956

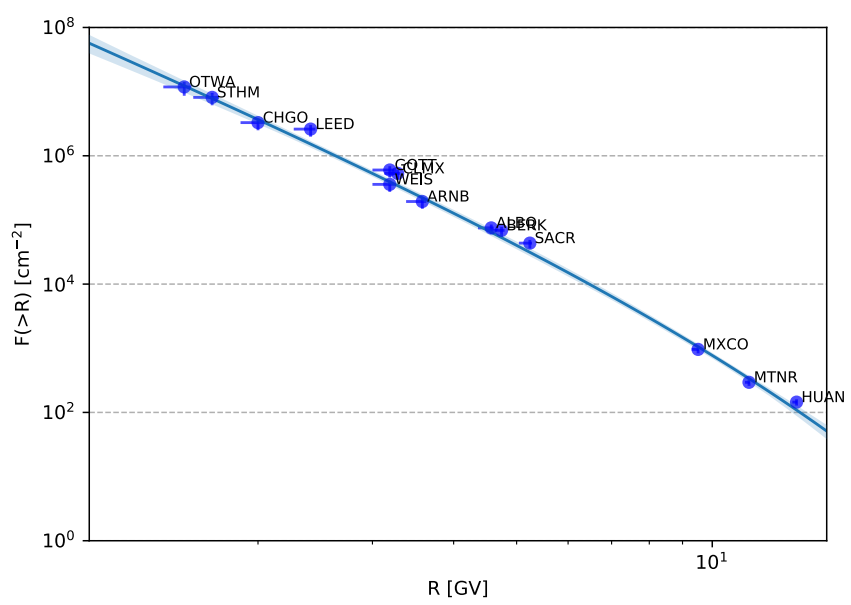

GLE10 // 12 Nov 1960

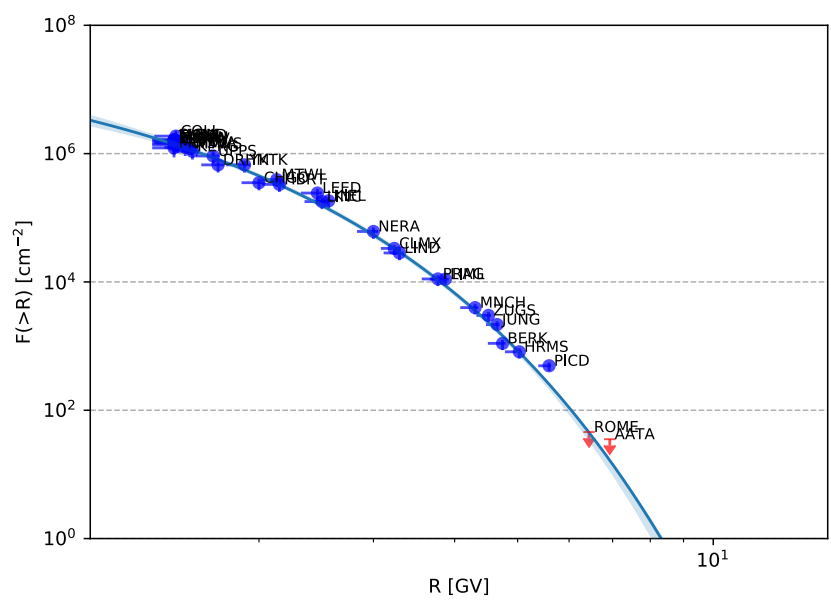

GLE12 // 20 Nov 1960

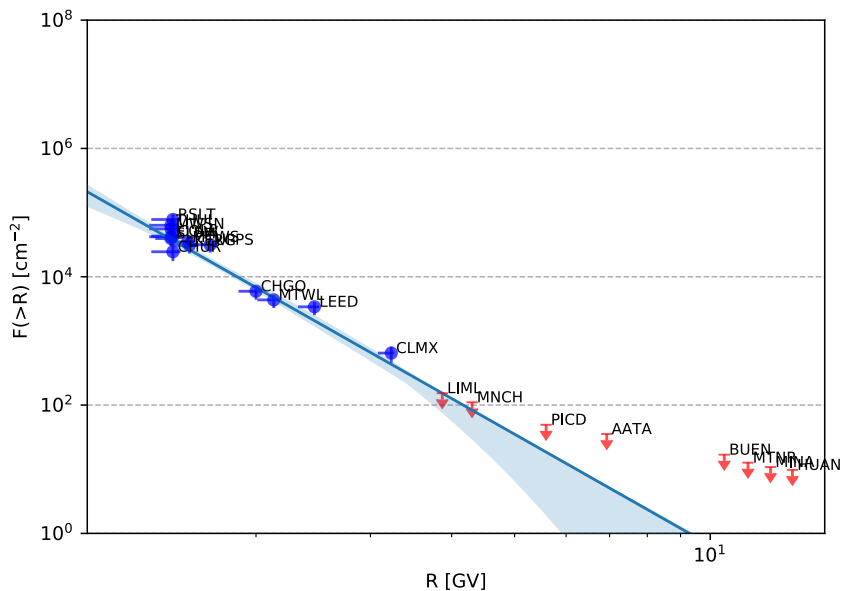

GLE08 // 04 May 1960

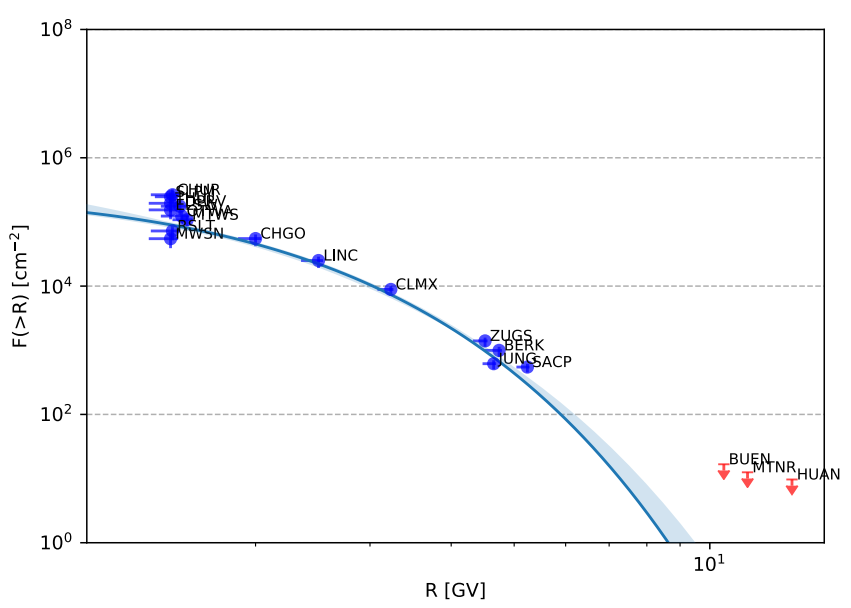

GLE11 // 15 Nov 1960

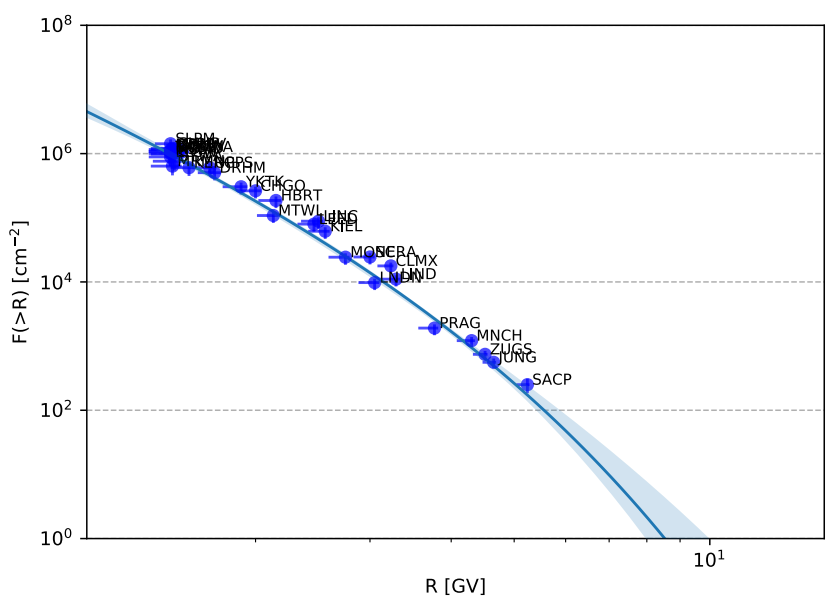

GLE13 // 18 Jul 1961

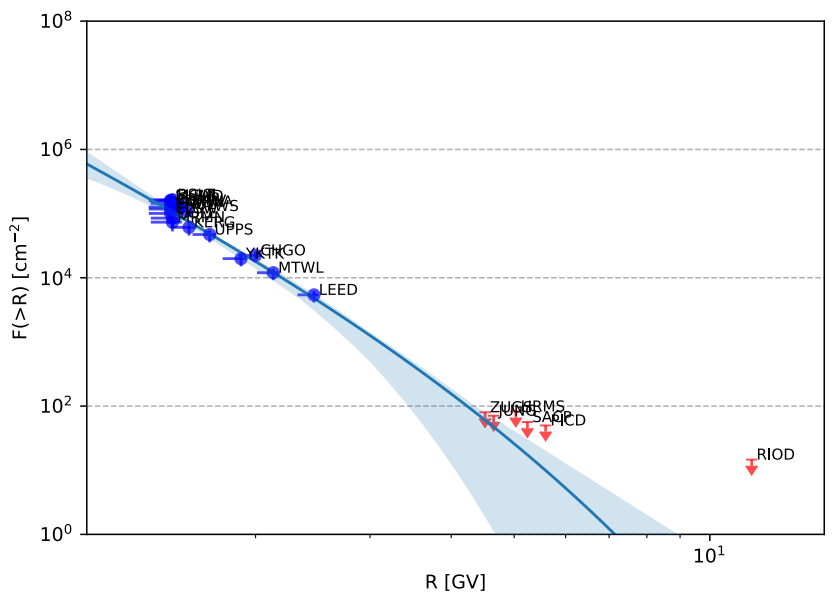

Fig. B.1. Integral fluences reconstructed here for all GLEs considered here (the GLE number and date are given in the header of each panel). Notations are similar as in Fig. 3 of the main text. 
GLE16 // 28 Jan 1967

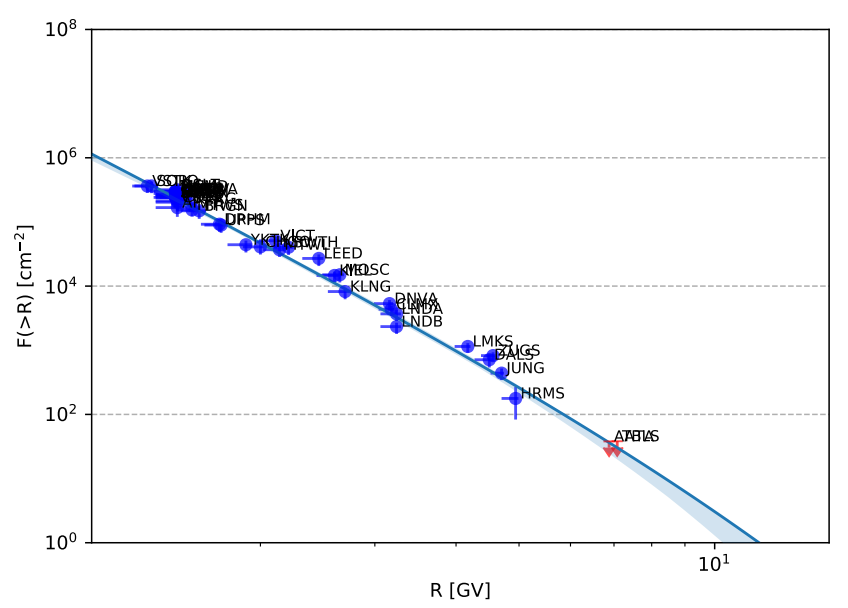

GLE19 // 18 Nov 1968

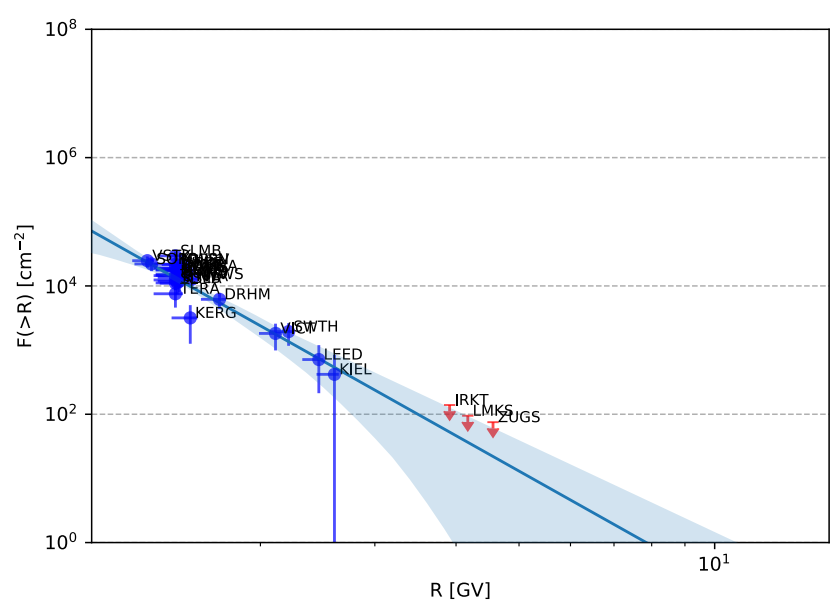

GLE21 // 30 Mar 1969

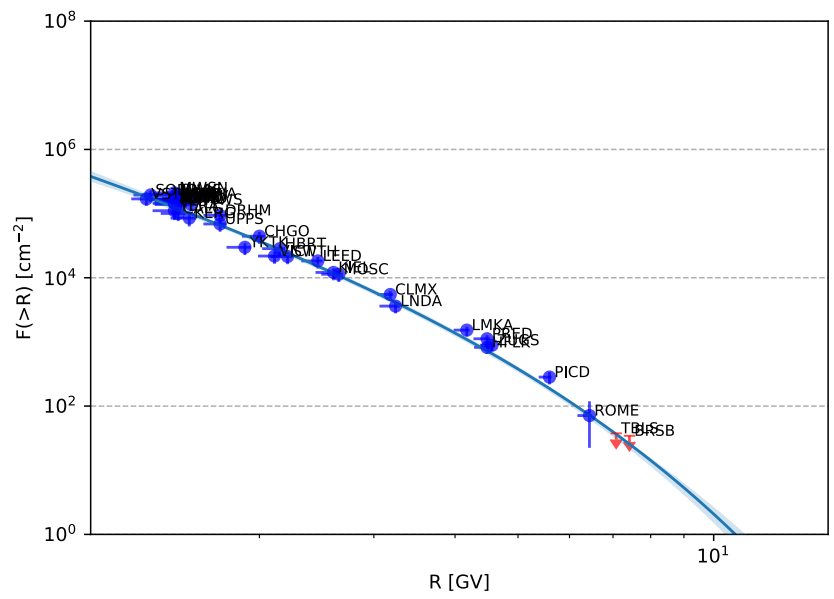

GLE18 // 29 Sep 1968

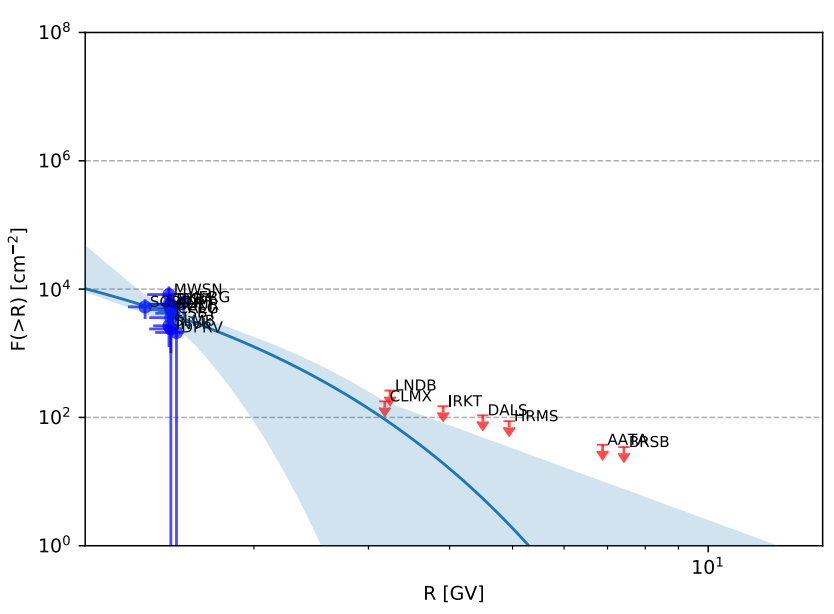

GLE20 // 25 Feb 1969

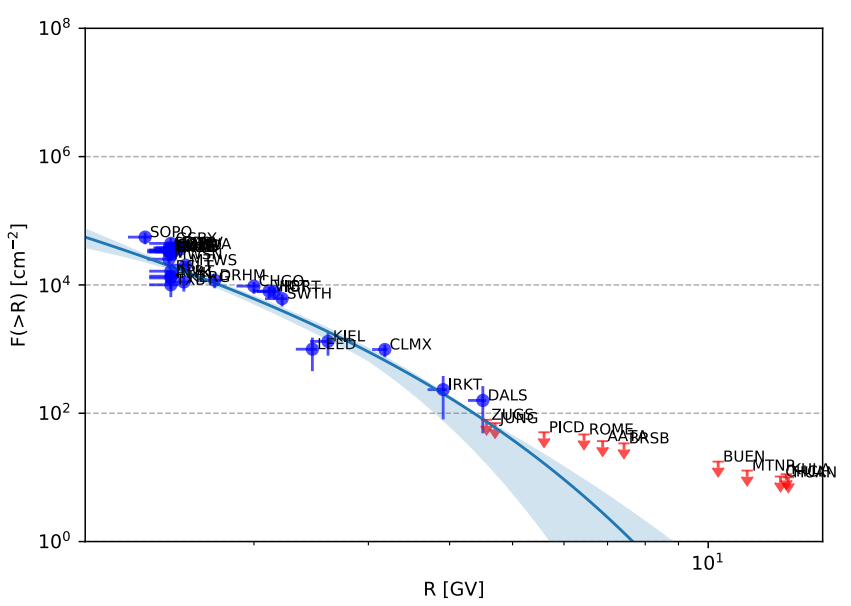

GLE22 // 24 Jan 1971

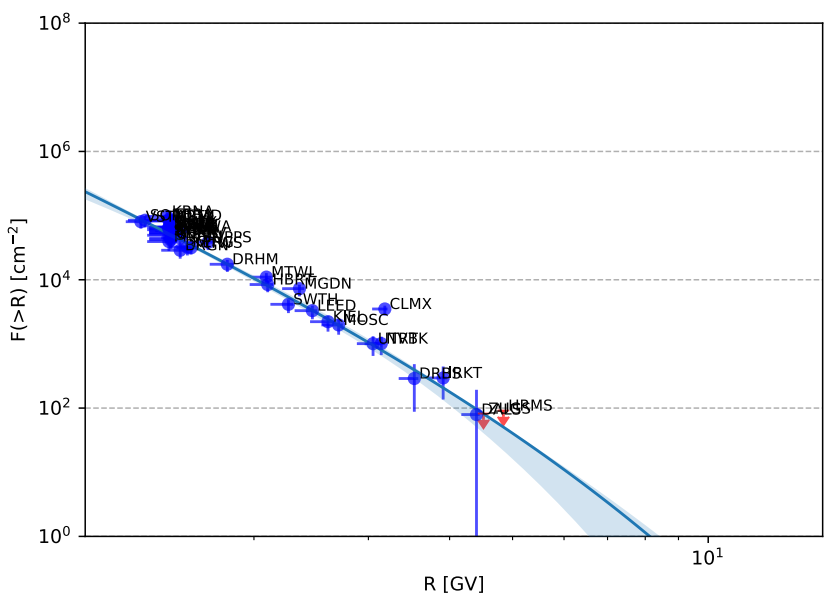

Fig. B.1. continued. 
GLE23 // 01 Sep 1971

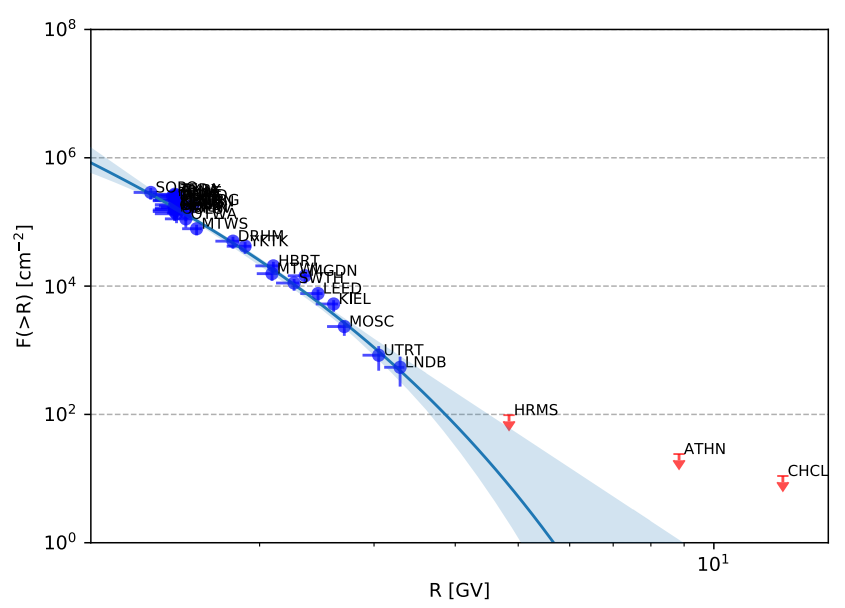

GLE25 // 07 Aug 1972

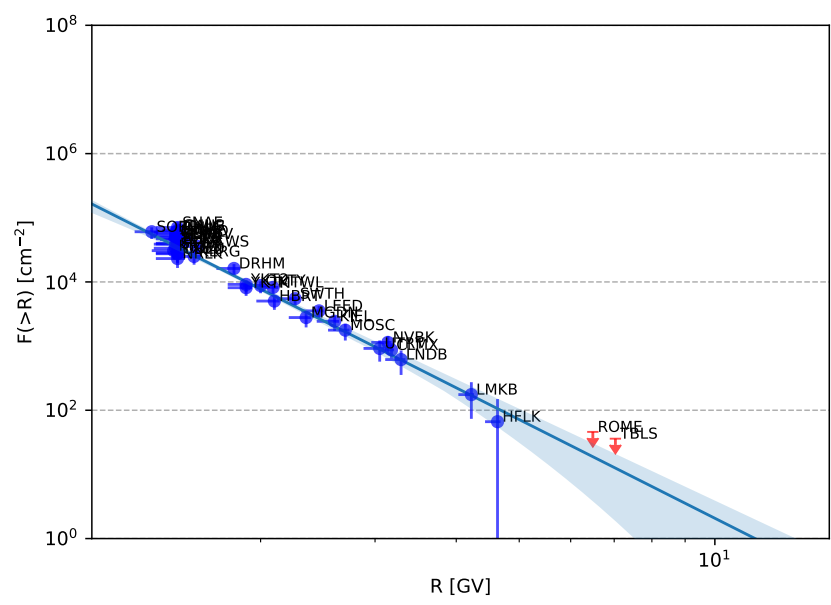

GLE27 // 30 Apr 1976

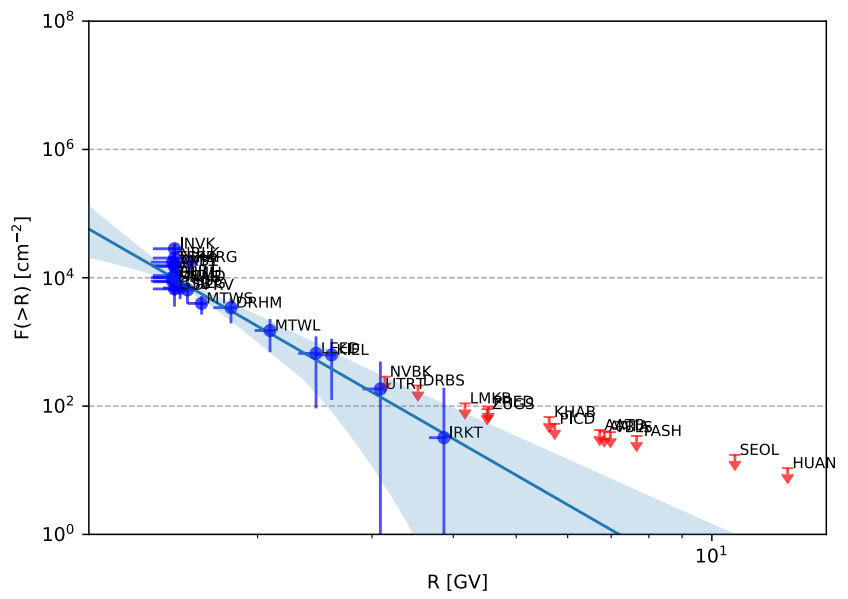

GLE24 // 04 Aug 1972

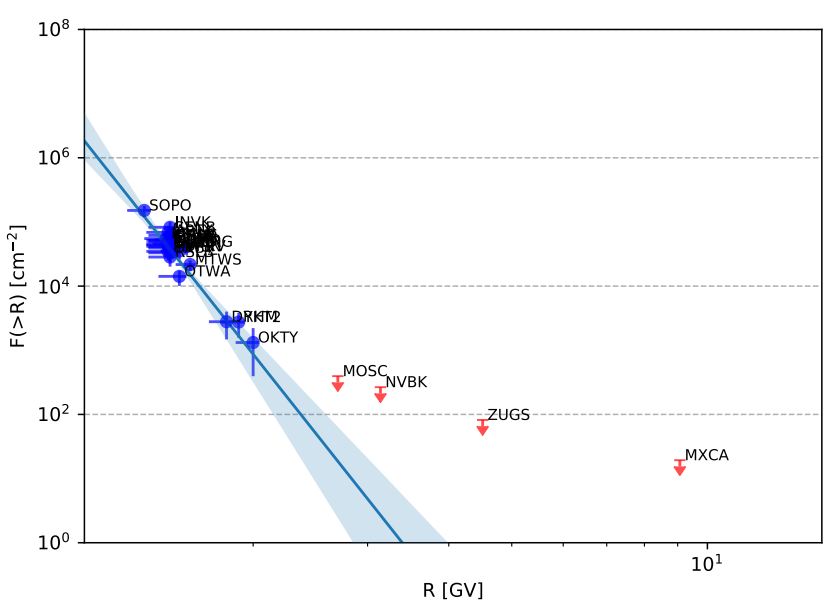

GLE26 // 29 Apr 1973

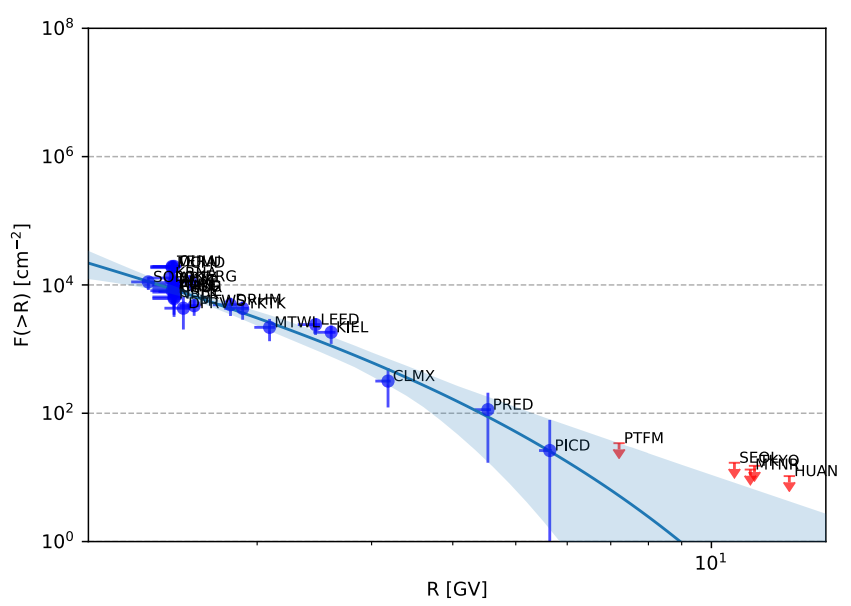

GLE28 // 19 Sep 1977

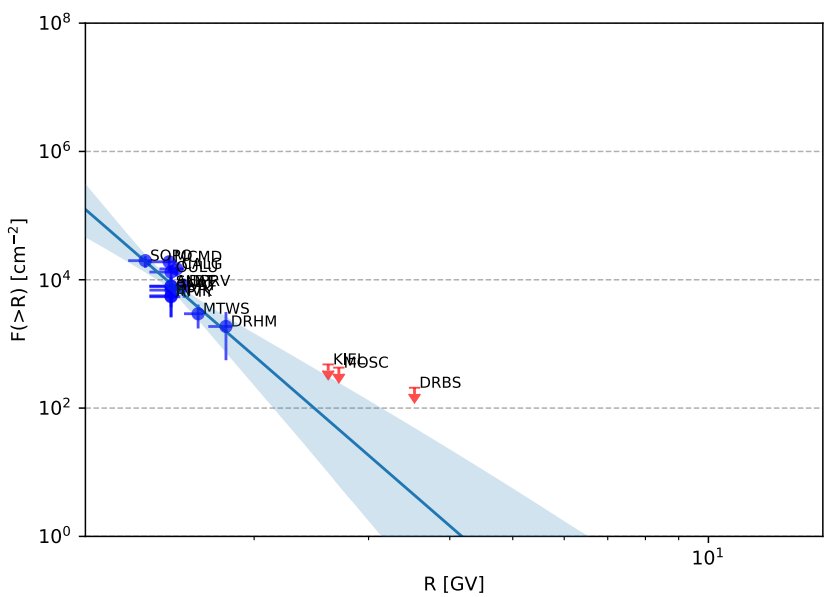

Fig. B.1. continued. 
GLE29 // 24 Sep 1977

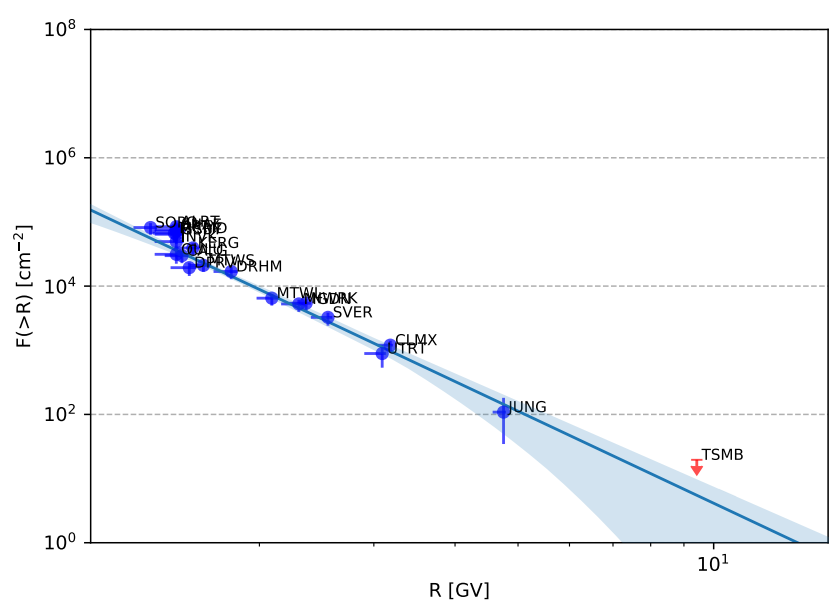

GLE31 // 07 May 1978

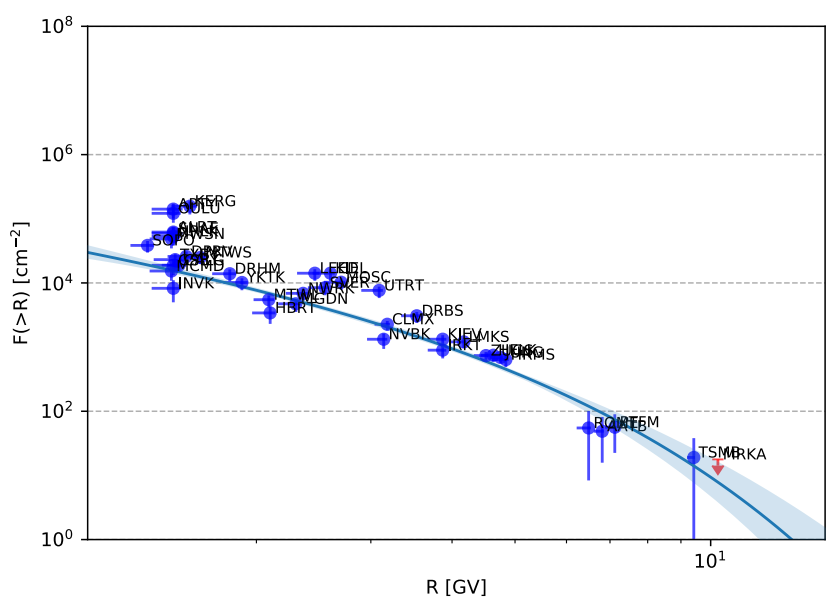

GLE33 // 21 Aug 1979

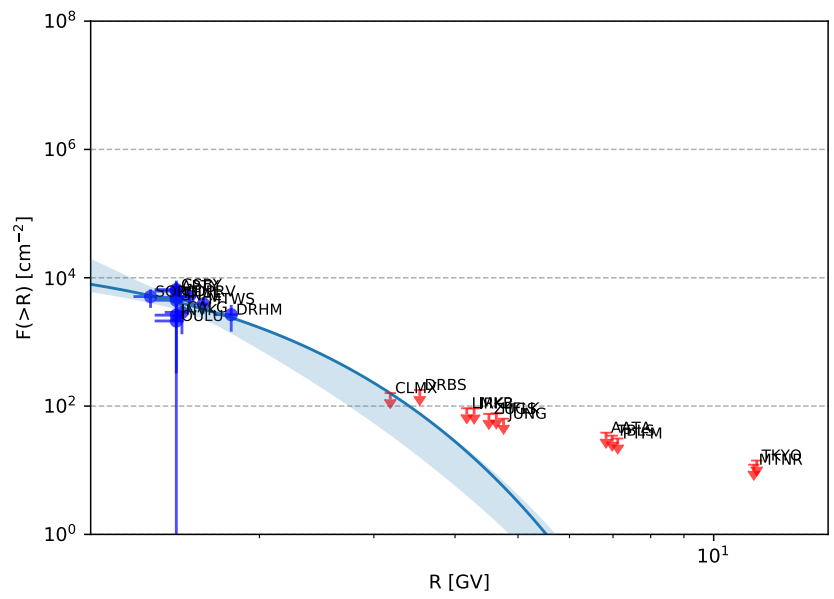

GLE30 // 22 Nov 1977

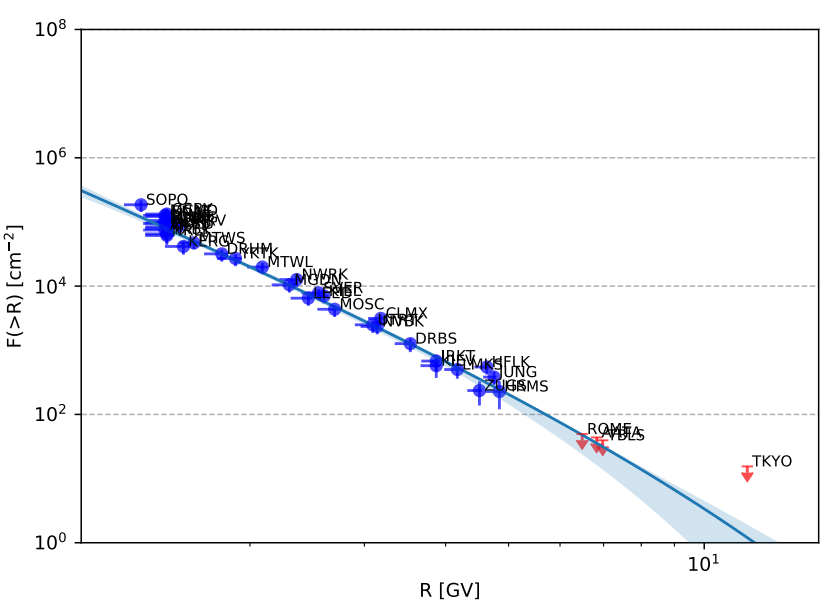

GLE32 // 23 Sep 1978

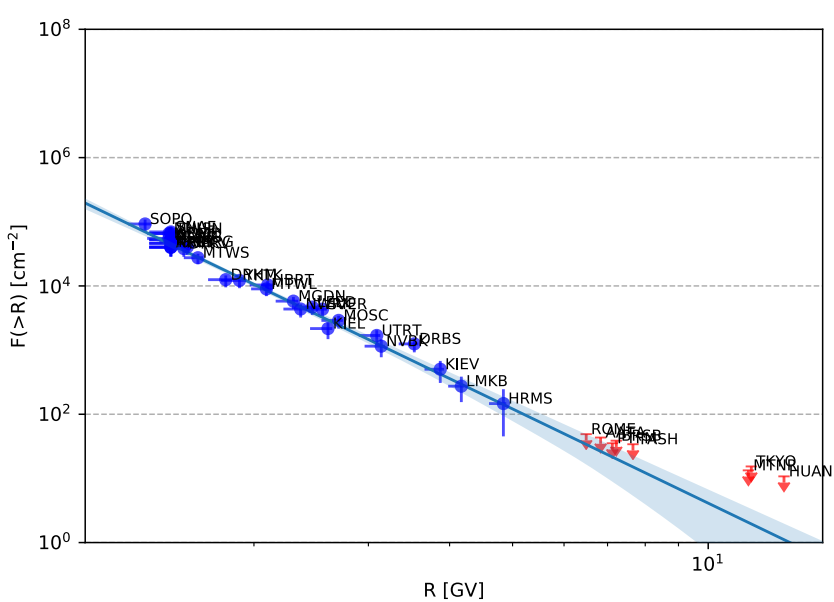

GLE35 // 10 May 1981

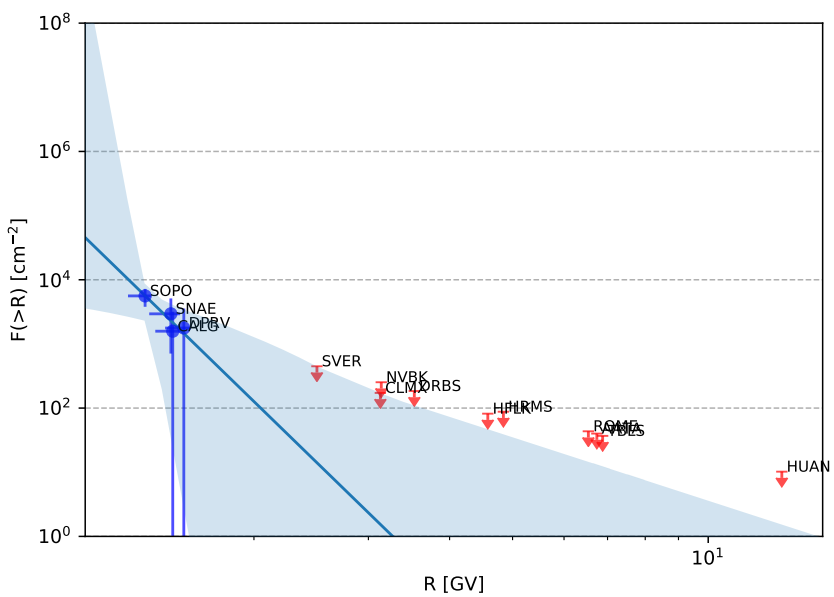

Fig. B.1. continued. 
GLE36 // 12 Oct 1981

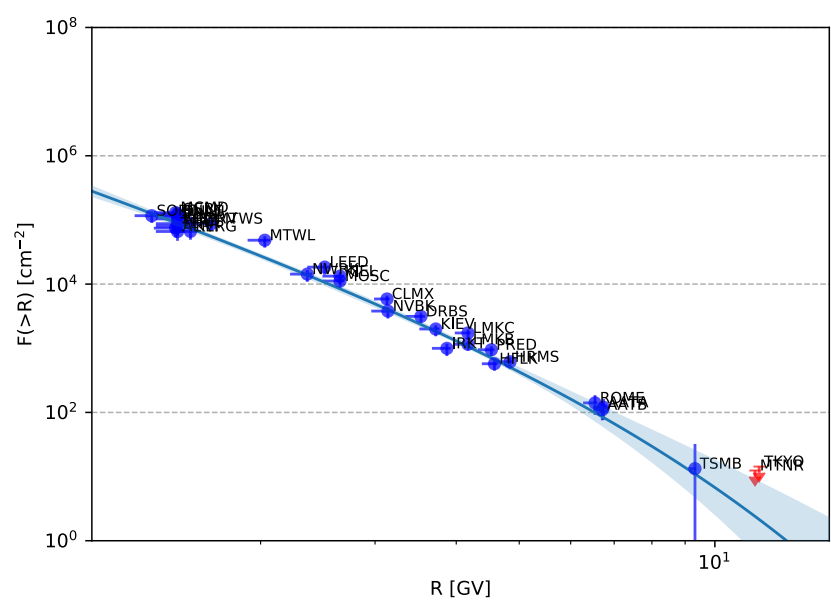

GLE38 // 07 Dec 1982

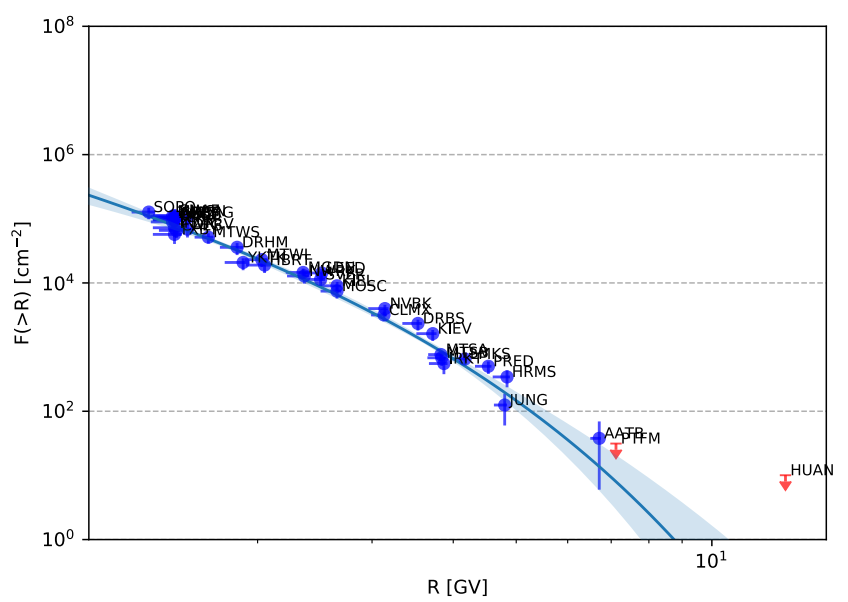

GLE40 // 25 Jul 1989

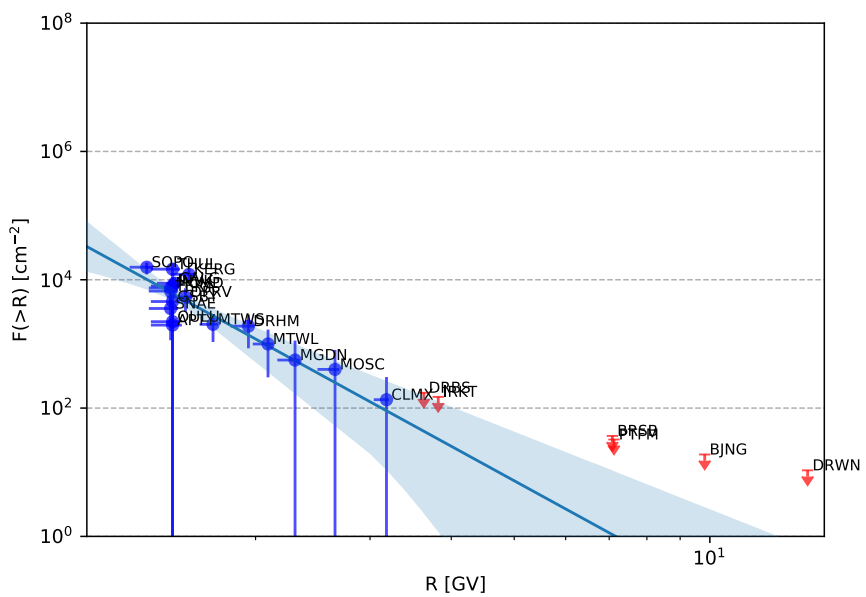

GLE37 // 26 Nov 1982

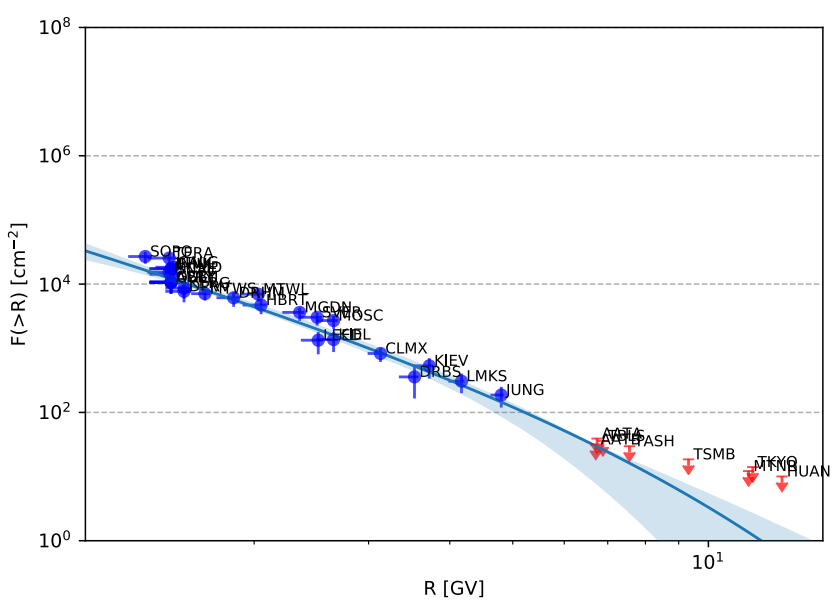

GLE39 // 16 Feb 1984

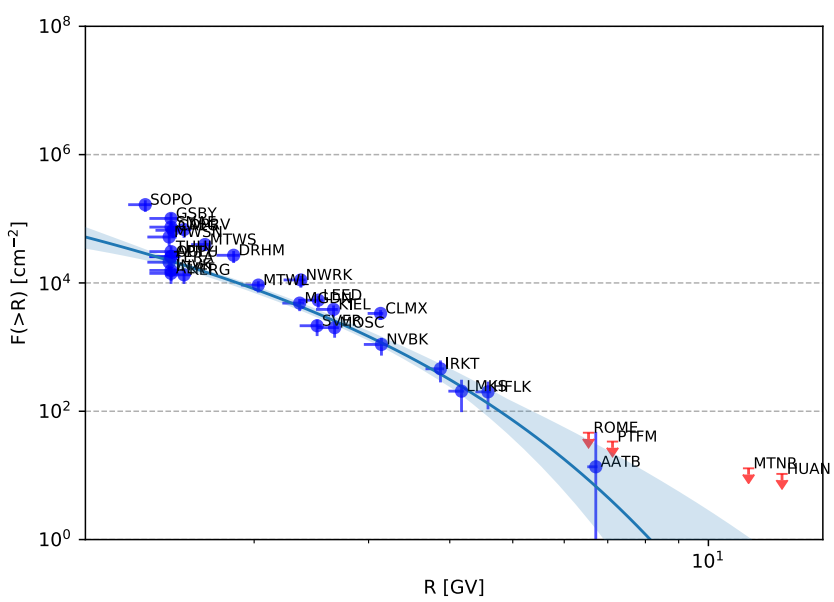

GLE41 // 15 Aug 1989

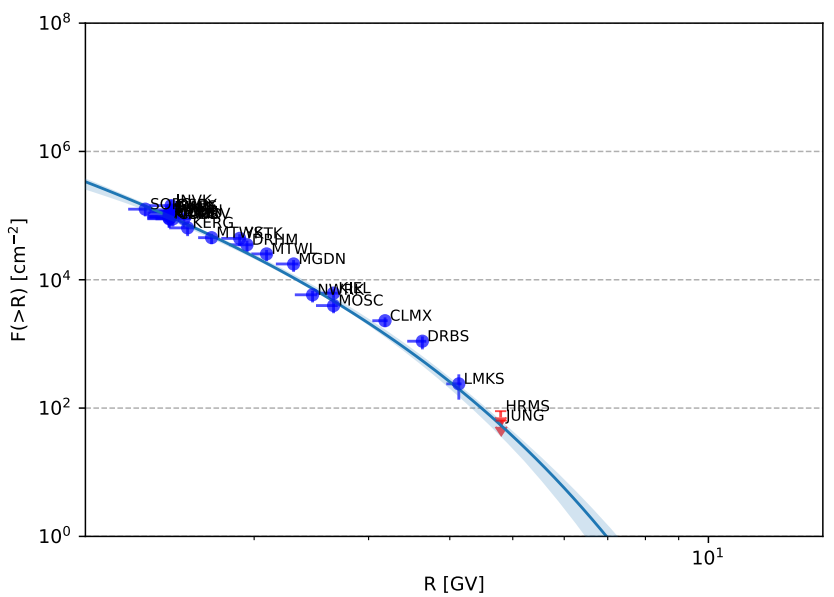

Fig. B.1. continued. 
GLE42 // 29 Sep 1989

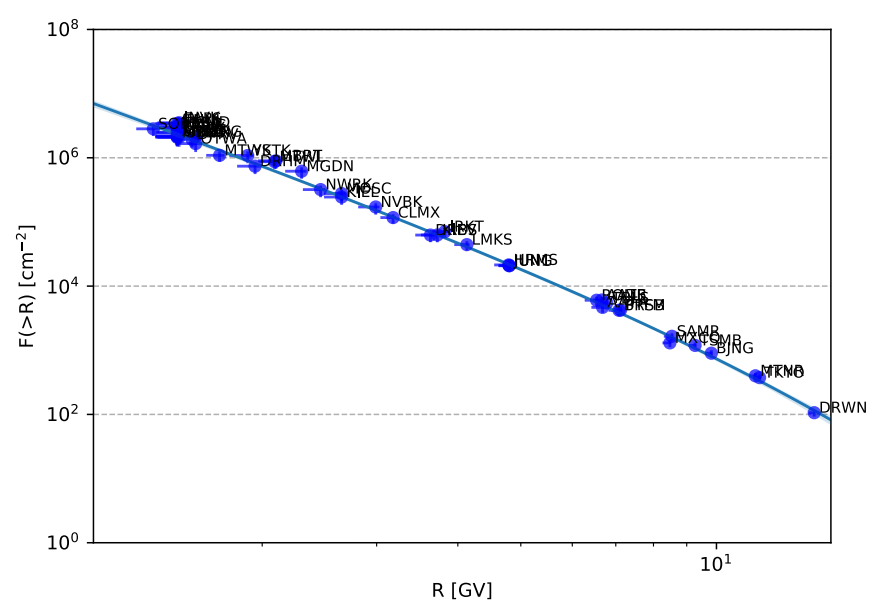

GLE44 // 22 Oct 1989

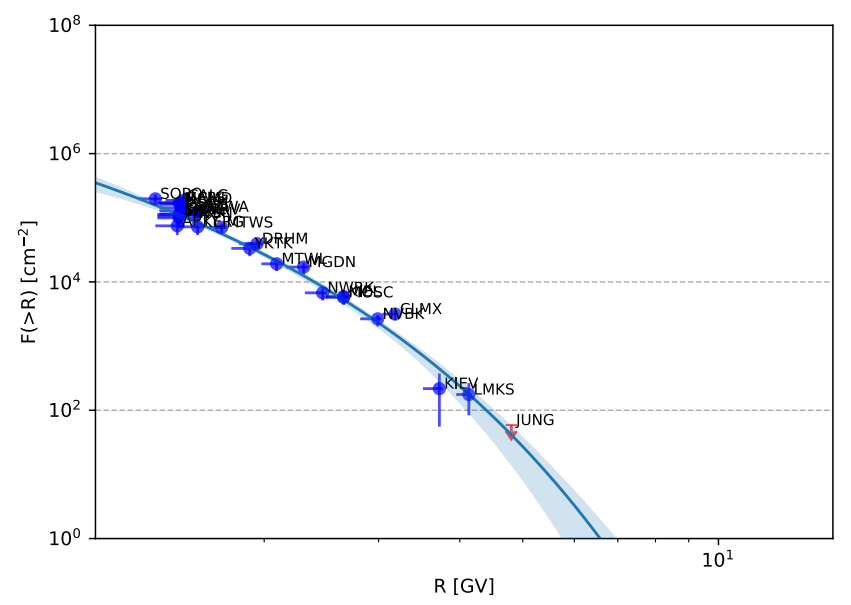

GLE46 // 15 Nov 1989

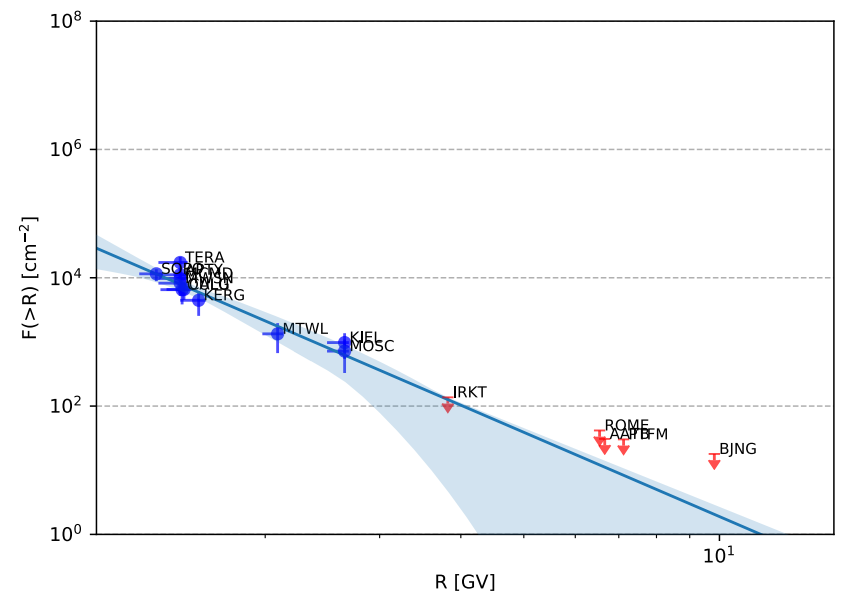

GLE43 // 19 Oct 1989

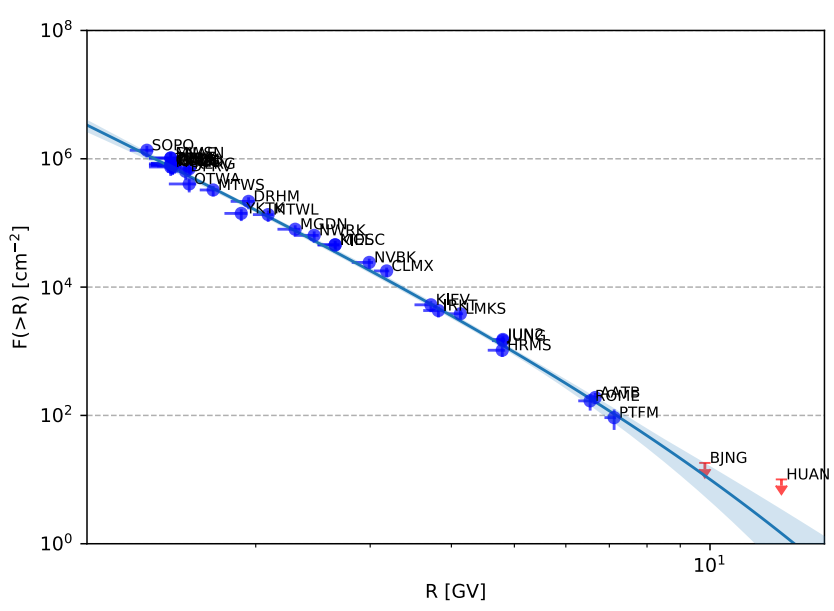

GLE45 // 24 Oct 1989

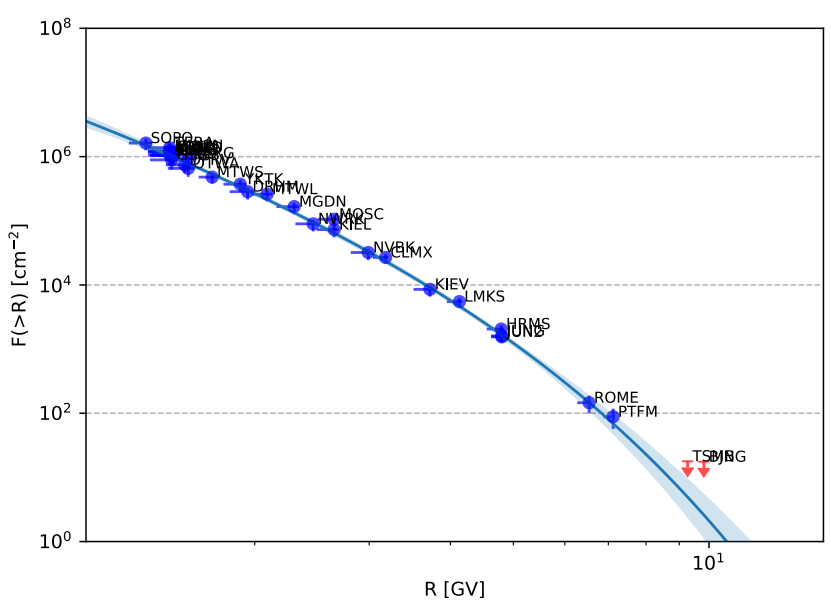

GLE47 // 21 May 1990

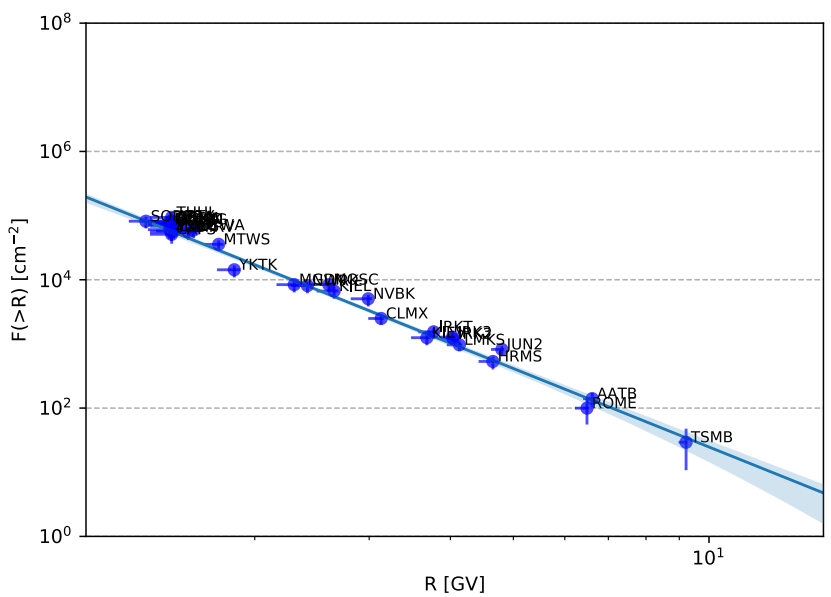

Fig. B.1. continued. 
GLE48 // 24 May 1990

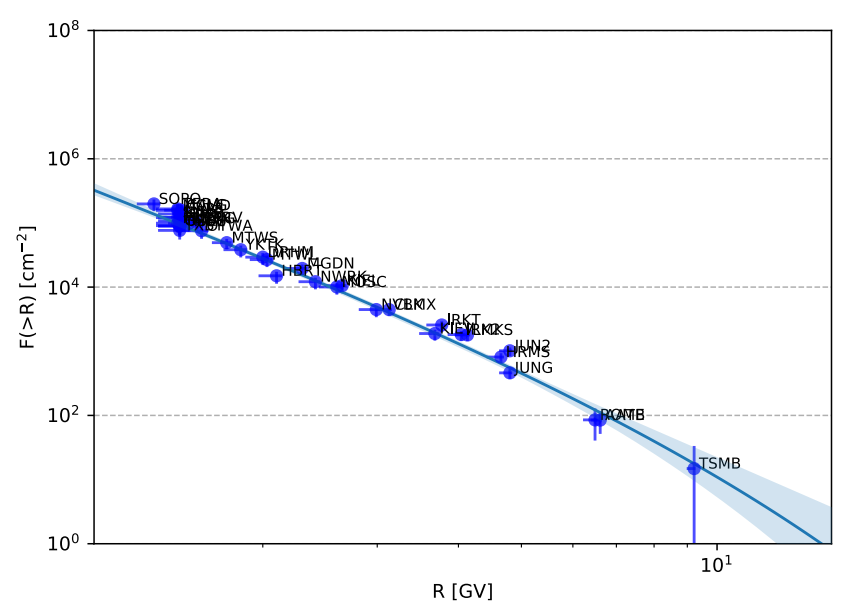

GLE50 // 28 May 1990

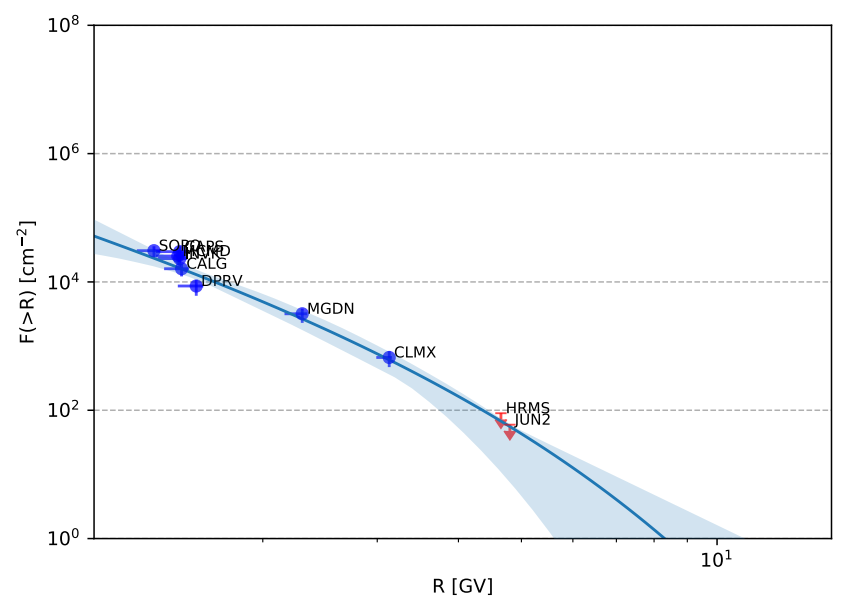

GLE52 // 15 Jun 1991

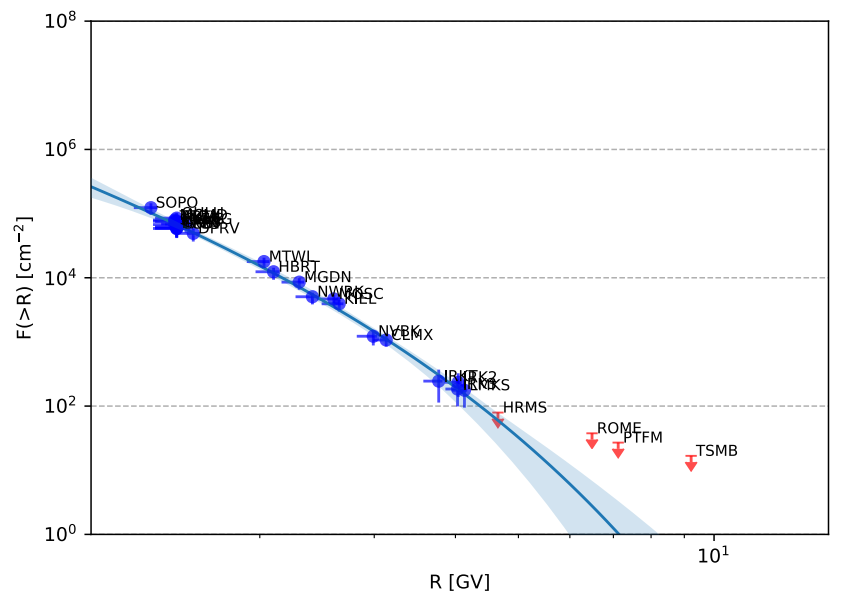

GLE49 // 26 May 1990

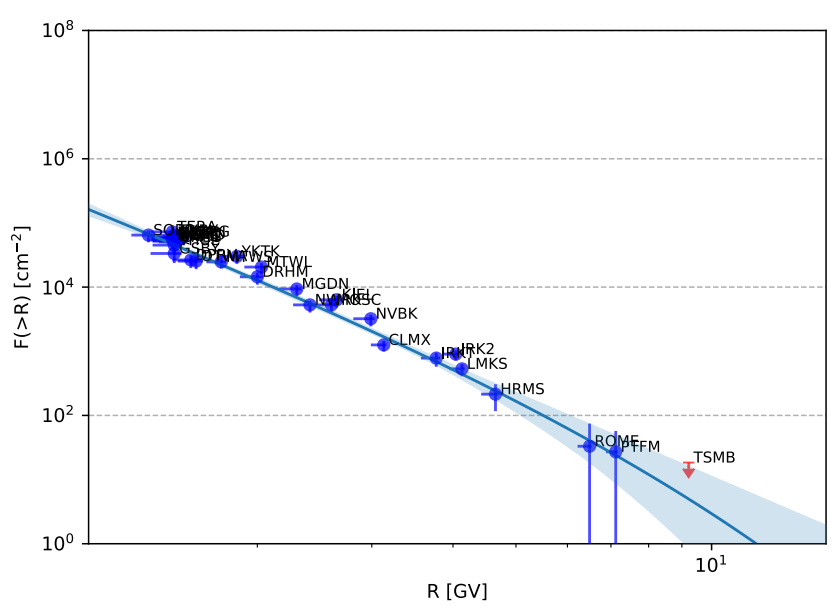

GLE51 // 11 Jun 1991

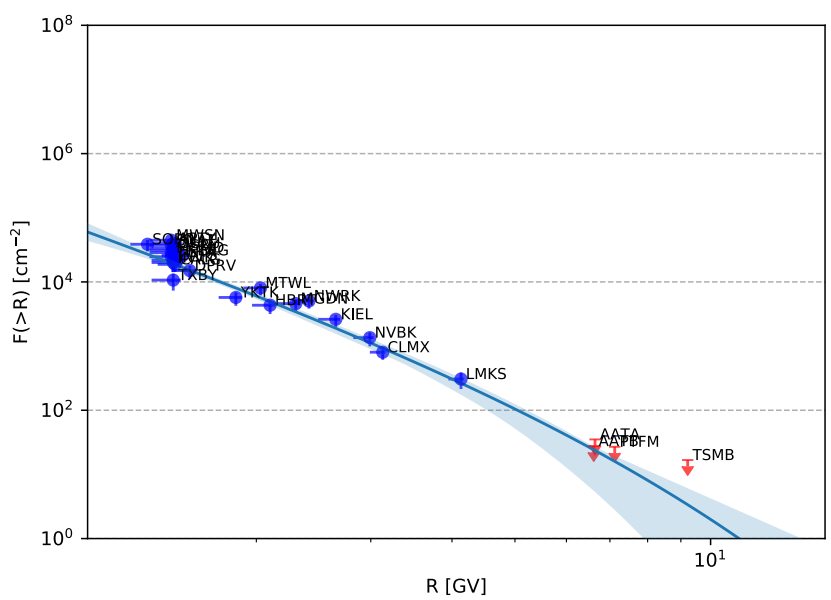

GLE53 // 25 Jun 1992

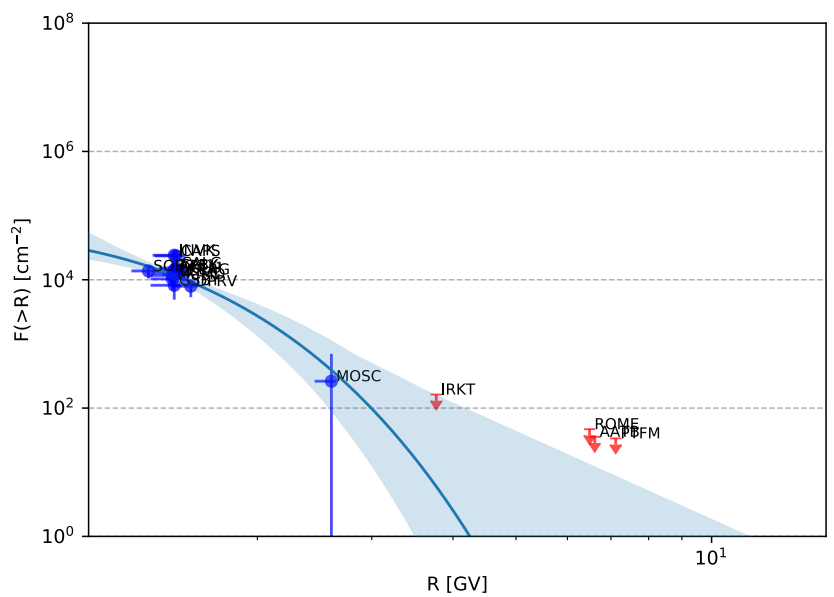

Fig. B.1. continued. 
GLE55 // 06 Nov 1997

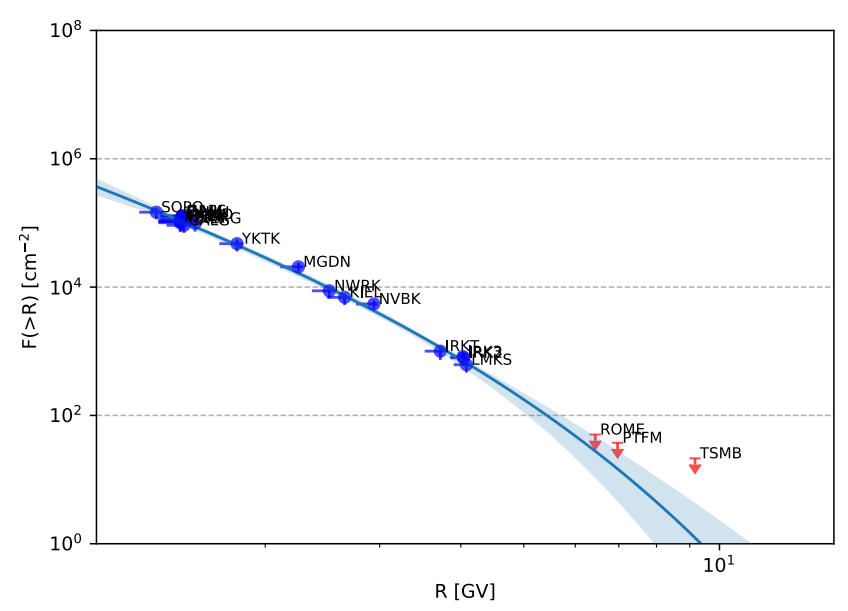

GLE58 // 24 Aug 1998

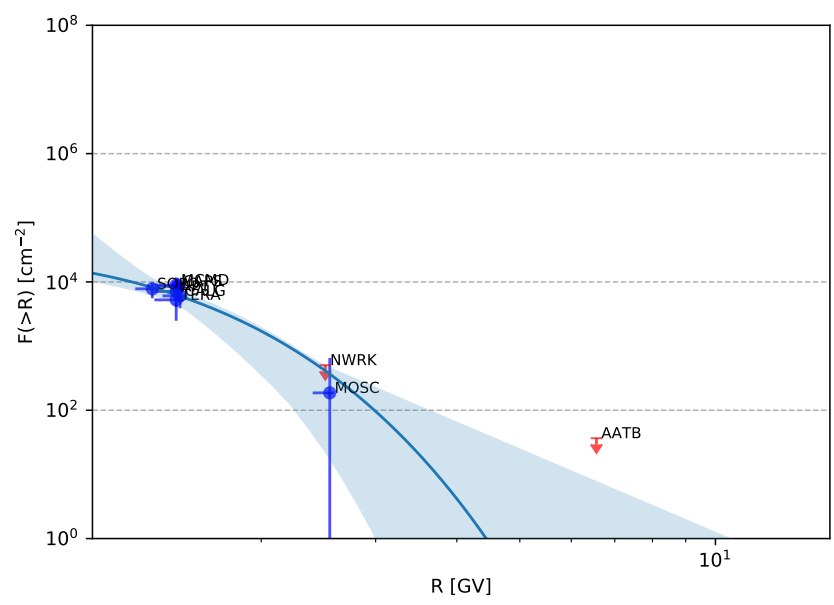

GLE60 // 15 Apr 2001

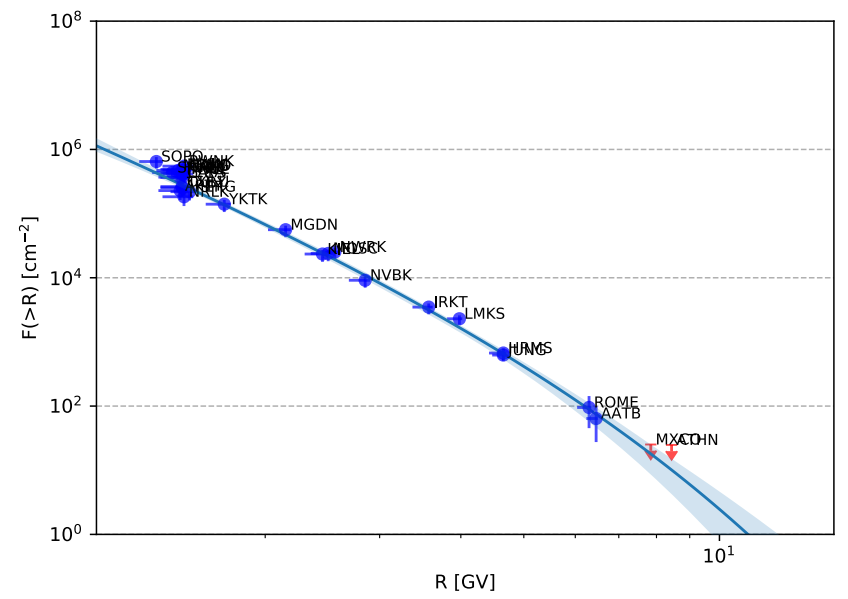

GLE56 // 02 May 1998

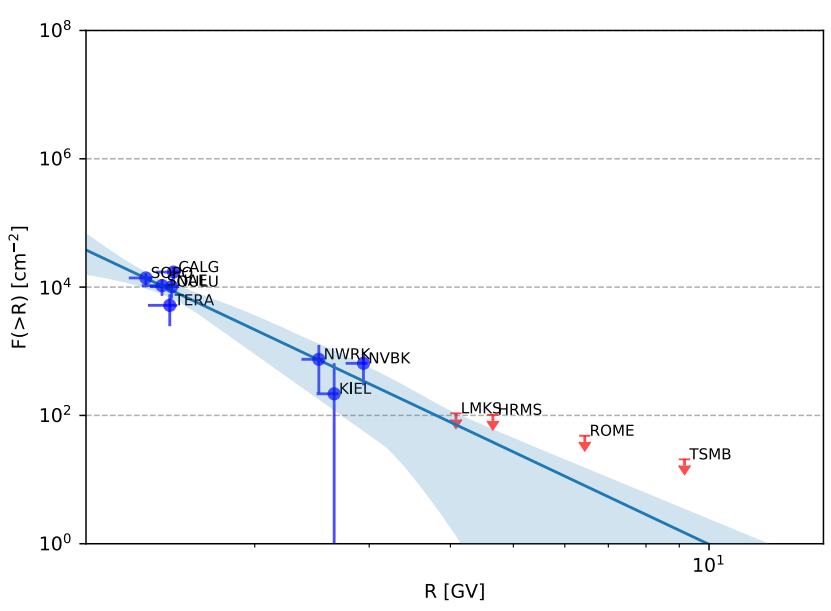

GLE59 // 14 Jul 2000

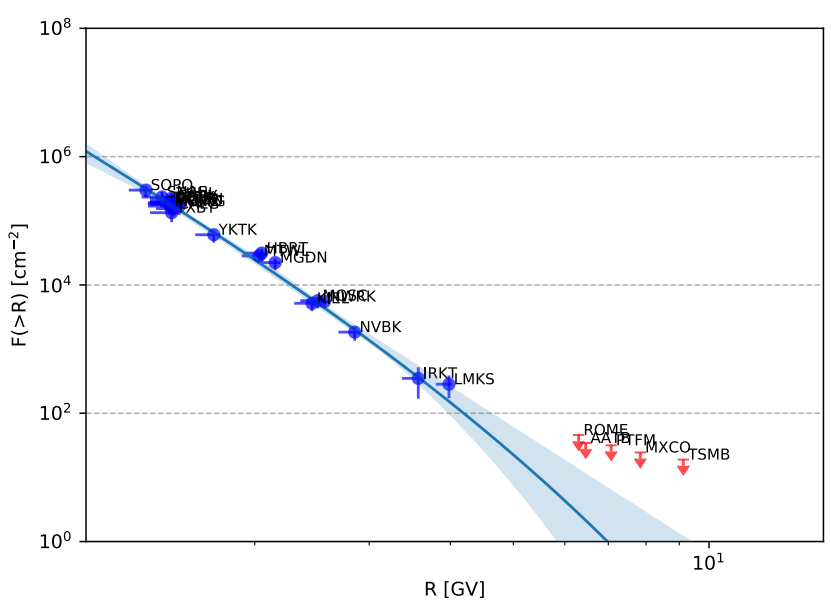

GLE61 // 18 Apr 2001

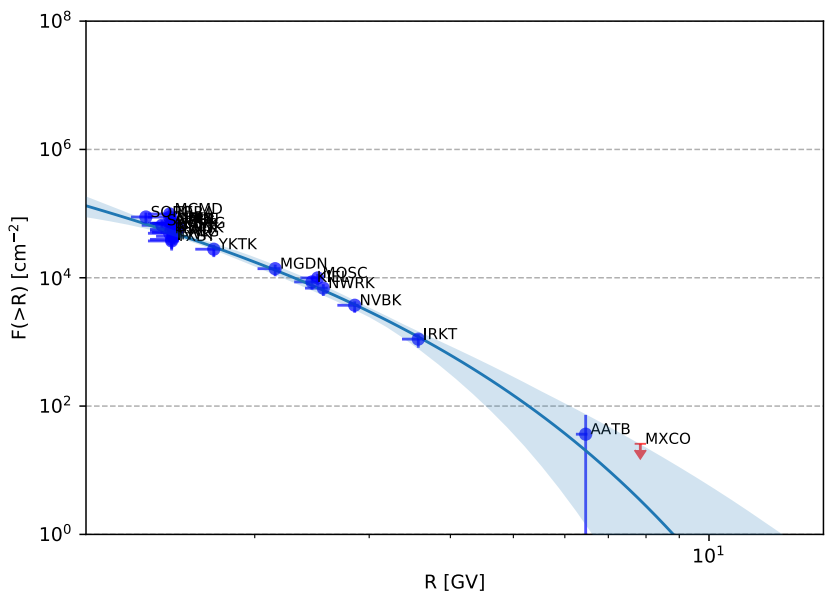

Fig. B.1. continued. 
GLE62 // 04 Nov 2001

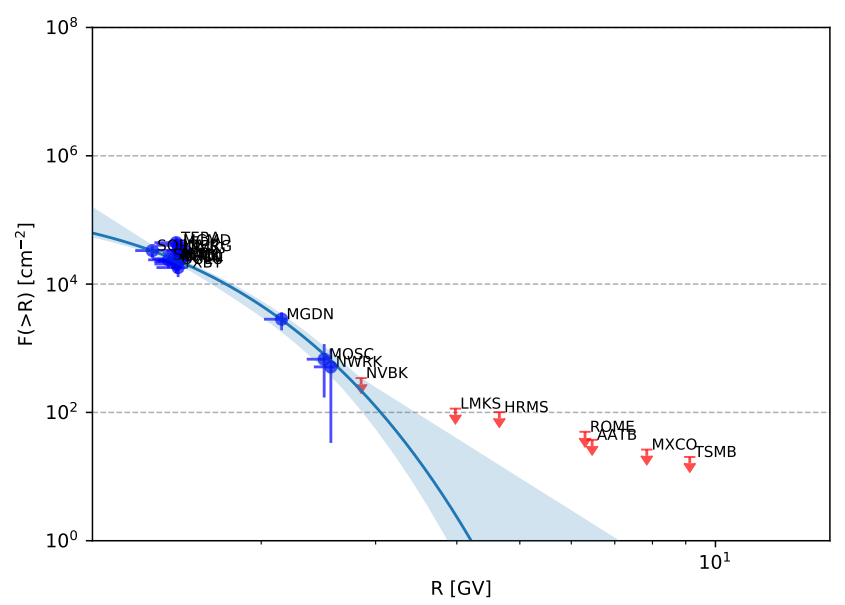

GLE64 // 24 Aug 2002

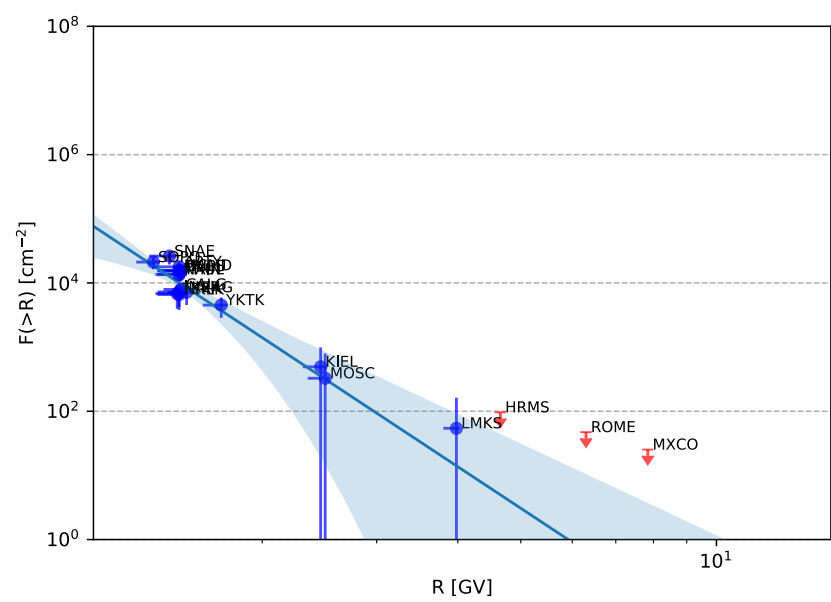

GLE66 // 29 Oct 2003

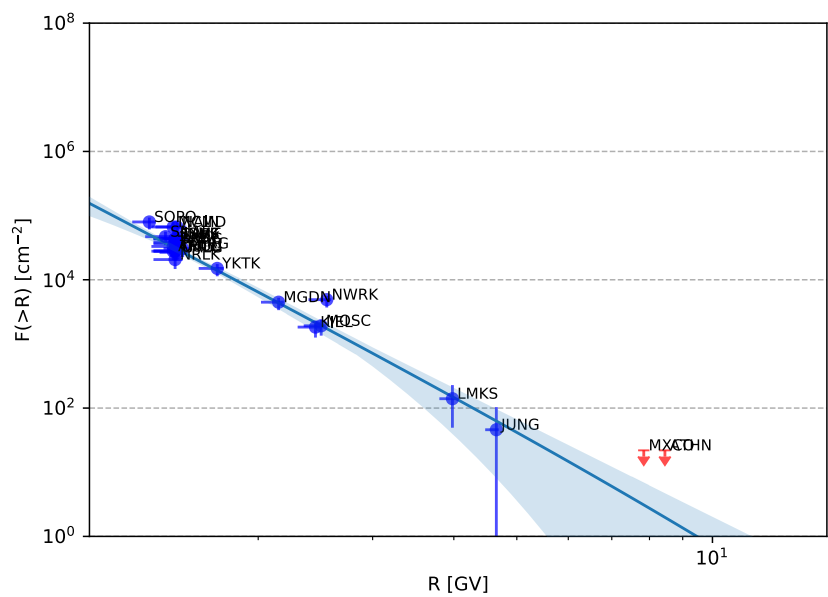

GLE63 // 26 Dec 2001

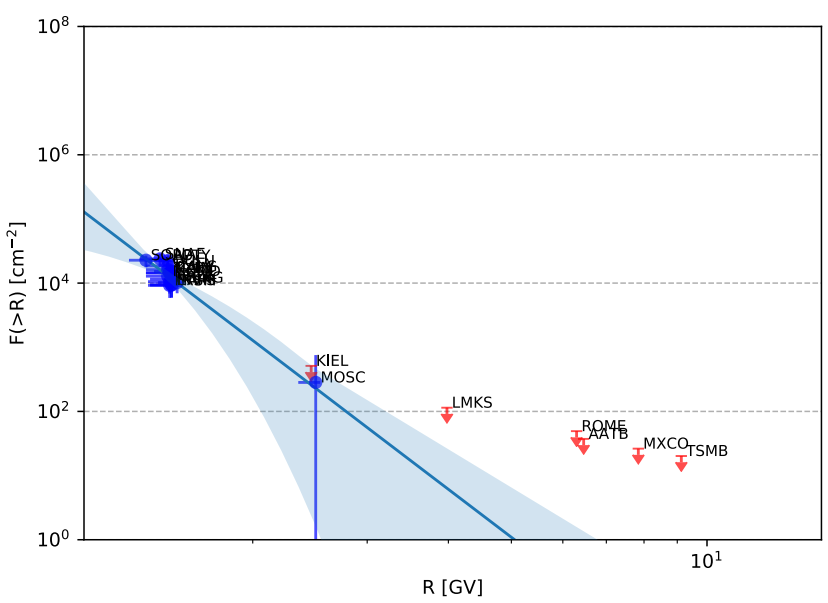

GLE65 // 28 Oct 2003

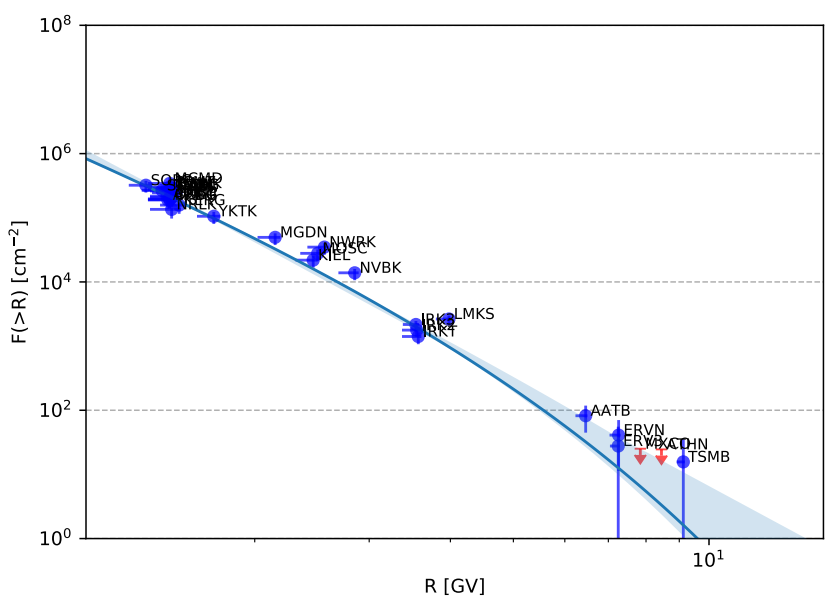

GLE67 // 02 Nov 2003

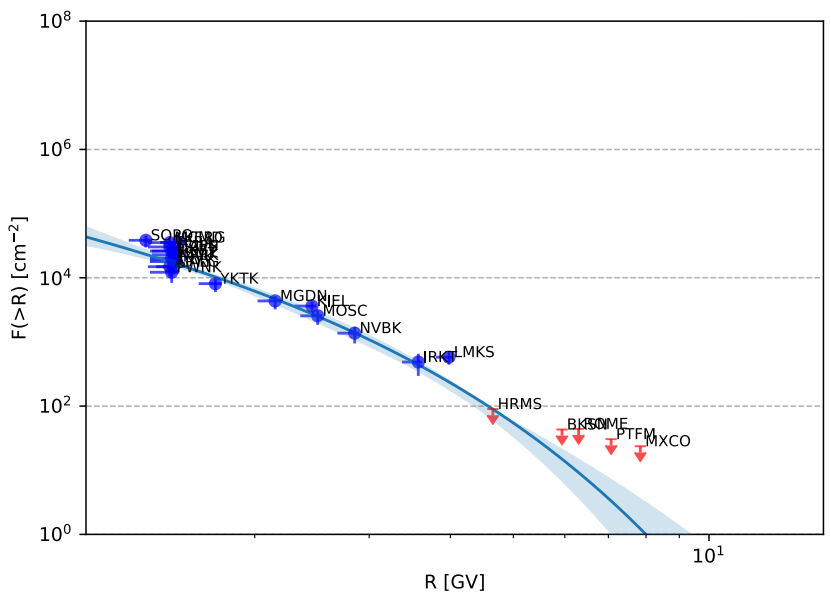

Fig. B.1. continued. 
GLE69 // 20 Jan 2005

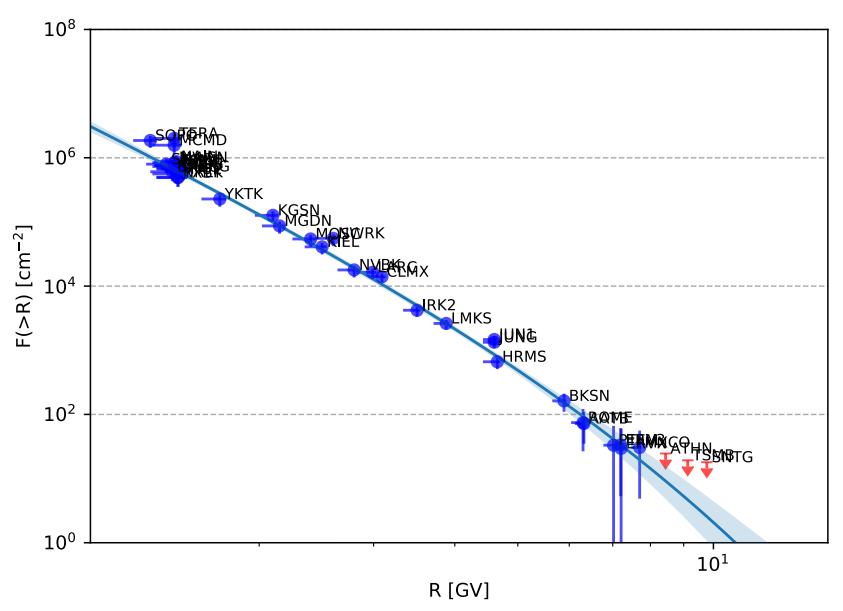

GLE71 // 16 May 2012

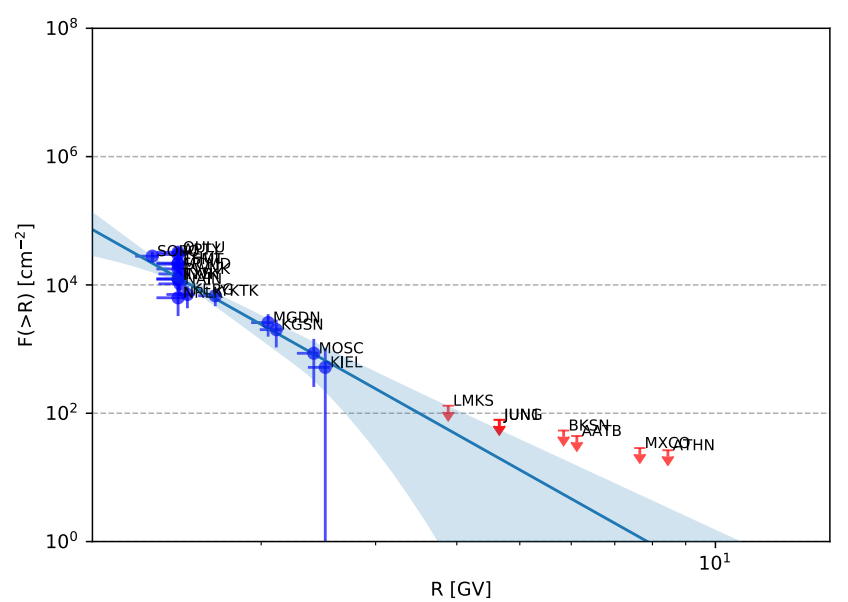

GLE70 // 13 Dec 2006

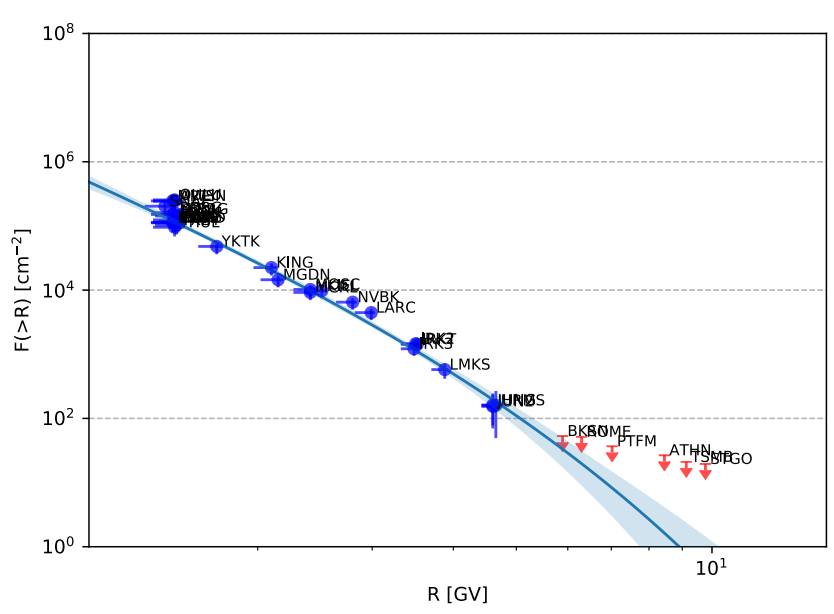

GLE72 // 10 Sep 2017

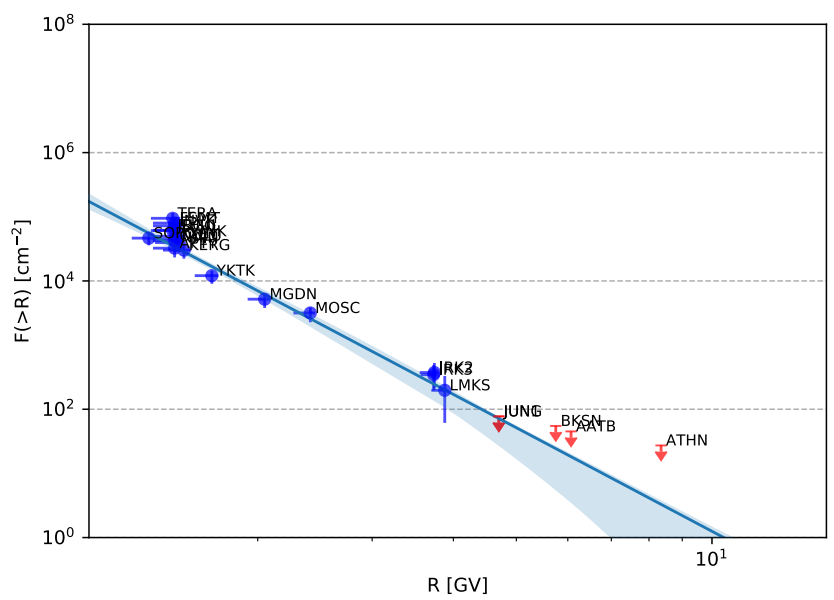

Fig. B.1. continued. 